MATHEMATICS OF COMPUTATION

Volume 74, Number 252, Pages 1707-1742

S 0025-5718(05)01745-X

Article electronically published on May 18, 2005

\title{
HETEROGENEOUS MULTISCALE METHODS FOR STIFF ORDINARY DIFFERENTIAL EQUATIONS
}

\author{
BJORN ENGQUIST AND YEN-HSI TSAI
}

\begin{abstract}
The heterogeneous multiscale methods (HMM) is a general framework for the numerical approximation of multiscale problems. It is here developed for ordinary differential equations containing different time scales. Stability and convergence results for the proposed HMM methods are presented together with numerical tests. The analysis covers some existing methods and the new algorithms that are based on higher-order estimates of the effective force by kernels satisfying certain moment conditions and regularity properties. These new methods have superior computational complexity compared to traditional methods for stiff problems with oscillatory solutions.
\end{abstract}

\section{INTRODUCTION}

We consider stiff ordinary differential equations (ODEs)

$$
\frac{d u_{\epsilon}}{d t}=f_{\epsilon}\left(u_{\epsilon}, t\right),
$$

where $u_{\epsilon}: \mathbb{R}^{+} \mapsto \mathbb{R}^{d}$, and assume that the eigenvalues $\lambda_{\epsilon}^{(j)}(t)$ of the Jacobian of $f_{\epsilon}$ satisfy the following conditions for $t \geq 0$ : 1) $\operatorname{Re} \lambda_{\epsilon}^{(j)} \leq C_{1}, 1 \leq j \leq d$; 2) there is $k_{0} \geq 1$ such that $\left|\lambda_{\epsilon}^{(j)}\right| \leq C_{2}$, for $1 \leq j \leq k_{0} \leq d$ and $C_{3} \leq \epsilon\left|\lambda_{\epsilon}^{(j)}\right| \leq C_{4}$, for $k_{0}<$ $j \leq d$; here $C_{1}, C_{2}, C_{3}$, and $C_{4}$ are constants; 3$) \min _{j_{1}, j_{2}}\left|\lambda_{\epsilon}^{\left(j_{1}\right)}(t)-\lambda_{\epsilon}^{\left(j_{2}\right)}(t)\right|>\rho>0$, $j_{1} \leq k_{0}$ and $j_{2}>k_{0}$.

A linear equation of this sort can be written as

$$
\frac{d u_{\epsilon}}{d t}=A_{\epsilon}(t) u_{\epsilon}+\phi(t)
$$

where

$$
A_{\epsilon}(t)=S(t)\left(\begin{array}{cc}
\epsilon^{-1} A^{I}(t) & 0 \\
0 & A^{I I}(t)
\end{array}\right) S^{-1}(t),
$$

$S, S^{-1}, A^{I}$ and $A^{I I}$ are bounded independent of $\epsilon,\left|\sigma\left(A_{\epsilon}^{I}\right)\right|>\delta>0, \operatorname{Re}\left(\sigma\left(A_{\epsilon}^{I}\right)\right) \leq 0$, and $|d \phi / d t|$ is bounded independent of $\epsilon$.

We will also consider the nonlinear model system $u_{\epsilon}(t)=(x(t), y(t))$ that takes the form:

$$
\left\{\begin{array}{l}
\epsilon \dot{x}=f^{I}(x, y, t), \\
\dot{y}=f^{I I}(x, y, t),
\end{array}\right.
$$

Received by the editor July 31, 2003 and, in revised form, June 4, 2004.

2000 Mathematics Subject Classification. Primary 65Lxx, 65Pxx, 37Mxx.

The second author is partially supported by the National Science Foundation under agreement No. DMS-0111298. 
where $f^{I}$ and $f^{I I}$ are smooth functions. We call $y$ the slow variable of the system.

1.1. Description of methods. The numerical methods that we shall discuss in this paper are devised under the HMM (Heterogeneous Multiscale Methods) framework [6]. We first present the general structure of the proposed methods and then relate them to other existing work.

Assume that there exists an "effective" system

$$
\frac{d}{d t} U=\bar{f}(U, t)
$$

that is derived from (1.1) as $\epsilon$ converges to 0 , such that the partial derivatives of $\bar{f}$ are bounded independent of $\epsilon$. Our methods construct solutions to (1.4) by evaluating the right-hand side of (1.4) "on the fly" via numerical solutions to (1.1). The explicit form of $\bar{f}$ is not used in the algorithm, and the precise definition for (1.4) may depend on the explicit forms of (1.1) and on different applications.

For example, in the linear constant coefficient cases of (1.2), if

$$
A^{I}=\left(\begin{array}{cc}
-\alpha & 0 \\
0 & i \beta
\end{array}\right), \alpha>0, \beta \in \mathbb{R} \backslash\{0\},
$$

and

$$
A^{I I}=\gamma
$$

then

$$
\bar{f}=S\left(\begin{array}{ccc}
0 & 0 & 0 \\
0 & 0 & 0 \\
0 & 0 & \gamma
\end{array}\right) S^{-1}+S\left(\begin{array}{ccc}
0 & 0 & 0 \\
0 & 0 & 0 \\
0 & 0 & 1
\end{array}\right) S^{-1} \phi(t) .
$$

In nonlinear examples, the "effective" equations may come from averaging [1, 2. In the examples discussed in this paper, $U$ has the same dimension as $u_{\epsilon}$, and its components are the strong or weak limits of those of $u_{\epsilon}$. In more general settings, $U$ does not have to be in the same space as $u_{\epsilon}$.

A generic HMM method is described by the scheme (macro-solver) used to solve (1.4) for $U$, and another scheme (micro-solver) used to solve (1.1) for evaluating the missing data; i.e., the effective force $\bar{f}$. This structure is best illustrated by Figure 1.1 the upper directed axis represents the grid imposed by the macrosolver to hold the values of $U$, and the lower axis contains the finer grids on which the solutions of (1.1) are constructed by the micro-solver, with initial conditions $u_{\epsilon, n}\left(t_{n}\right)$ determined from the grid values of $U$. The downward pointing arrows symbolize the determination of $u_{\epsilon, n}\left(t_{n}\right)$ from $U$ at $t_{n}$. The upward pointing arrows relate the evaluation of $\bar{f}$ to the time history of microscale variables $u_{\epsilon, n}(t)$ and the forces $f_{\epsilon}\left(u_{\epsilon, n}(t)\right)$ that are obtained from each micro-grid on the bottom axes. This evaluation is accomplished through averaging with a compactly supported kernel $K$. We present the theory of kernels in Section 2.

A basic algorithm can be summarized by the following steps:

(1) Force estimation:

(a) At $T=t_{n}, u_{0}=U^{n}$.

(b) Solve

$$
\frac{d u_{\epsilon, n}}{d t}=f_{\epsilon}\left(u_{\epsilon, n}, t\right), \quad u_{\epsilon, n}\left(t_{n}\right)=u_{0},
$$

for $t \in\left[t_{n}, t_{n}+\eta\right]$.

(c) Averaging: $\bar{f}\left(t_{n}\right) \sim \tilde{f}\left(t_{n}\right)=K * f_{\epsilon}\left(u_{\epsilon, n}\right)$. 


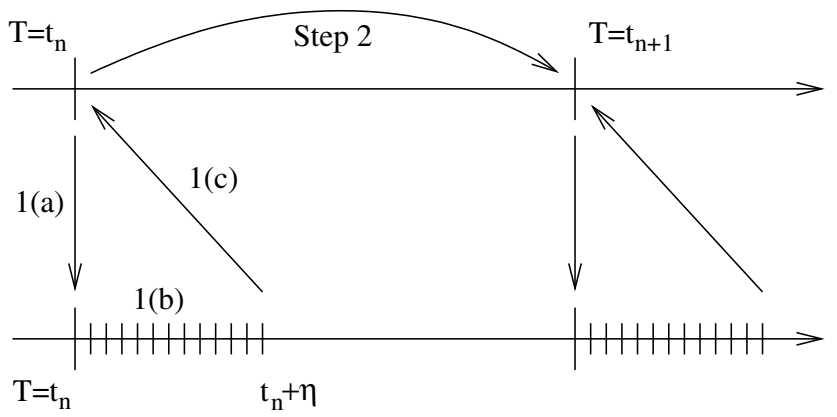

Figure 1.1. A basic HMM ode solver schematics

(2) Evolve the macro variables: compute $U^{n+1}$ at $T=t_{n+1}$ using $\left\{U^{j}\right\}_{j=0}^{n}$ and $\left\{\tilde{f}\left(t_{j}\right)\right\}_{j=0}^{n}$.

(3) Repeat.

As an example, a generic HMM multistep method can be arranged in the following form:

where $\tilde{f}$ is computed by

$$
\tilde{f}\left(U^{n}, t_{n}\right)=\sum_{j=0}^{2 m} h K\left(t_{n}, t_{j}\right) f_{\epsilon}\left(\mathcal{S}^{h}\left(t_{n}, t_{n}^{j}\right) U^{n}, t_{n}^{j}\right),
$$

where $t_{n}^{j}=t_{n}+j h$, and $\mathcal{S}^{h}\left(t_{0}, t_{1}\right)$ is the discrete solution operator defined by the micro-solver. Here, as well as in the remainder of this paper, we use $H$ and $h$ for the discrete time steps used in the macro- and micro-grid respectively.

In Sections 2 and 3, we will see that an HMM scheme can be analyzed systematically by examining the evaluations of the scheme on the macroscale grid

$$
\frac{d U}{d t}=\bar{f}(U, t) .
$$

The local error of the macroscale scheme contains the local truncation error of the macro-solver (Step 2) and the numerical and analytical errors of Step 1, i.e., the local error $E=\mathcal{E}_{\text {macro }}+\mathcal{E}_{H M M}$, where

$$
\mathcal{E}_{H M M}=\left|\bar{f}\left(U^{n}, t_{n}\right)-\mathcal{H}\left(f_{\epsilon}, U^{n}, t_{n}\right)\right|,
$$

and $\mathcal{H}\left(f_{\epsilon}, U^{n}, t_{n}\right)$ denotes Step 1 of the algorithm. At the end of Section 3, we will show the complexity of an HMM scheme through balancing $\mathcal{E}_{\text {macro }}$ and $\mathcal{E}_{H M M}$.

We call a method HMM-X-y if X-method is used in Step 2 and y-method is used in Step 1(b). Therefore, HMM-FE-rk4 is a method that uses forward Euler as macro-solver and a fourth-order Runge-Kutta method for micro-solver. In Section 3, we will present a few standard HMM schemes and discuss their stability in detail.

1.2. Generalizations. We notice from the basic algorithm above that $\bar{f}$ is approximated at $t_{n}$, which is the beginning time of each fine scale calculation in Step (b). In Section 2, we show that it is possible to select a kernel $K$ so that $\bar{f}$ is approximated at time $t_{n}+\delta t_{*}$. This is a key feature of our proposed algorithm. In 
many examples of this paper, we choose a kernel that is symmetric with respect to the center of its support and use $\delta t_{*}=\eta / 2$. The algorithm of [1] is similar to the case in which the Dirac delta function is used as the averaging kernel and $\bar{f}$ is evaluated at $t_{n}+\eta$. We remind the reader that $\eta$ denotes the length of each evolution performed in Step 1(b).

Here, we present a more complete algorithmic description below:

(1) Force estimation:

(a) Reconstruction: at $T=t_{n}, u_{0}=R U^{n}$.

(b) Solve

$$
\frac{d u_{\epsilon, n}}{d t}=f_{\epsilon}\left(u_{\epsilon, n}, t\right), \quad u_{\epsilon, n}\left(t_{n}\right)=u_{0},
$$

for $t \in\left[t_{n}, t_{n}+\eta\right]$.

(c) Averaging:

(i) Estimate force:

$$
\bar{f}\left(t_{n}+\delta t_{*}\right) \sim \tilde{f}\left(t_{n}+\delta t_{*}\right)=K * f_{\epsilon}\left(u_{\epsilon, n}\right) .
$$

(ii) Compression:

$$
U^{*}=Q\left[u_{\epsilon, n}\right] .
$$

(2) Evolve the macro variables: compute $U^{n+1}$ at $T=t_{n+1}$ using $\left\{U^{j}\right\}_{j=0}^{n}$, $\left\{\tilde{f}\left(t_{n}\right)\right\}_{j=0}^{n}$ and $U^{*}, \bar{f}\left(t_{n}+\delta t_{*}\right)$.

(3) Repeat.

Notice that Step 1(a) and Step 1(c) are changed a bit from the previous section. In this paper, the reconstruction operator $R$ will be taken to be the identity operator, i.e. $R U^{n}=U^{n}$, and the compression $Q\left[u_{\epsilon, n}\right]=u_{\epsilon, n}\left(t_{n}+\delta t_{*}\right)$. In [32], we apply the HMM ODE methods to a class of specific problems for which $R$ is no longer the identity operator.

There is a benefit in evaluating $\bar{f}$ at the center of each microscopic evaluation; it makes it possible to use a symmetric kernel that typically yields more accurate approximations to the averages. In many problems, such as the ones involving Hamiltonian systems, it is possible to evolve the given microscopic equation backward in time. In Step 1(b), one can instead obtain $u_{\epsilon, n}$ in $\left[t_{n}-\eta / 2, t_{n}+\eta / 2\right]$ and compute $\tilde{f}$ at $T=t_{n}$. Figure 1.2 illustrates the structure of two such schemes.

In the rest of this paper, we will refer to the steps of the algorithms described in this subsection.

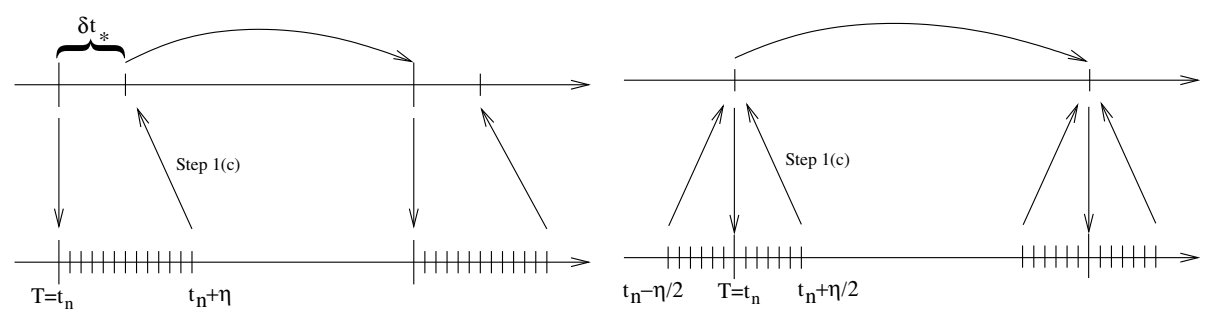

(a)

FiguRE 1.2. HMM ode solver schematics. 
1.3. Related work. A detailed review of numerical methods for stiff problems can be found in the books of Wanner et al [13, 14, and in a review paper 29].

There are essentially two types of stiff problems for which the solutions vary on the $\epsilon$ time scale $\left(\lambda_{\epsilon}^{(j)}=\mathcal{O}\left(\epsilon^{-1}\right)\right)$ : one is dissipative with rapid transients $\left(\operatorname{Re} \lambda_{\epsilon}^{(j)}<0\right)$ and the other is oscillatory in nature $\left(\operatorname{Re} \lambda_{\epsilon}^{(j)}=0\right)$. There are two standard approaches to such problems - numerical approximations and analytic techniques. Analytical techniques, such as perturbation and homogenization methods, typically require extensive algebraic manipulations and suffer from a limited applicability. Our focus will be on numerical approximation methods to which the main challenges come from the cost associated with maintaining the accuracy and stability of the approximation schemes for a time interval independent of $\epsilon$. In the following, we first briefly review the common methods related to each type of problem and compare their computational complexities to that of HMM methods. Finally, we comment on some other approaches that are not easily categorized.

Problems in the first class $\left(\operatorname{Re} \lambda_{\epsilon}^{(j)}<0\right)$ include, for example, chemical reactions systems. Implicit methods, such as BDF (backward difference formula) and IRK (implicit Runge-Kutta), are among the conventional choice of numerical solutions. There are also special explicit methods with variable step sizes that are designed to optimize the computation for special types of problems. In [21], Lebedev and Finogenov proposed an iterative method with variable time steps for dissipative systems whose eigenvalues cannot be separated into disjoint groups of different scales. Similar methods can also be found in, e.g., [4 and recently in [11] by Gear and Kevrekidis for stiff dissipative systems whose eigenvalues are well separated into two groups. The latter is called the projective integrator method and has been a source of inspiration for part of our present work.

Many problems in atmospheric science [17, molecular dynamics, biology, celestial mechanics, and circuit simulations fall into the oscillatory class. The complexity of explicit methods such as the Runge-Kutta or Leapfrog methods requires a step size of order $\epsilon$ for stability. Hence computing a solution of (1.1) to the final time $T_{1}$ requires at least order $\epsilon^{-1}$ operations. Implicit methods such as IRK or BDF can achieve optimal complexity for stiff dissipative problems since the $\epsilon$ order step sizes are only required at transients. However, when these methods are applied to oscillatory problems, typically convergence requires $\mathcal{O}\left(\epsilon^{-1}\right)$ time steps.

The methods developed in this paper offer solutions to the oscillatory problems as well as the dissipative ones. The gain in computational complexity emerges when the cost of evaluating $\bar{f}$ (i.e., the total time duration for each micro-evolution at the lower axes in Figure 1.1) is bounded above by $C \epsilon^{-\alpha}$ for some $\alpha<1$. We first notice that, structurally, our proposed algorithms share similarities to the multirevolutionary methods [10, 12, 24, or the quasi-envelope method 28]. In these methods, $\bar{f}$ is estimated by following the oscillations for a few periods. However, these methods are not adequate for problems with unknown periodicity of oscillations that consist of more than one frequency. We shall see in this paper that the proposed methods do not require precise information of the periodicities of oscillations, but nevertheless give accurate estimation of $f$. This comes from our time-averaging approach with smooth kernels.

The idea of averaging of the forces or the solutions is closely related to some perturbation techniques; see, e.g., 1, 2]. Computationally, averaging approaches appeared in many places, e.g., in [27, 34] and [25, 26], and recently in [9] and [22]. 
We present an averaging theory that includes the known moment conditions and some additional "stiffness reducing" properties of the kernels that result in efficient computations. In this paper, we develop a theory for which kernels should be used in the evaluation of $\bar{f}$, and how long and how accurate each microscale evolution should be for a given class of equations, so that the proposed methods become more computationally efficient and flexible than the other conventional ones. We study the stability, convergence and complexity of our algorithms. Typically, the computational complexity for an HMM scheme is $\mathcal{O}\left(\epsilon^{-\gamma} H^{-1}\right)$ where $\gamma<1$ and $H$ is the step size for the macroscale variable $U$.

The HMM technique can also be related to the operator splitting schemes used in meteorology [3] or to the mollified impulse scheme for Hamiltonian dynamics [9]. Some might even find a resemblance to the multirate methods, e.g. [10] and 22. However, in these methods, the stiffness that comes from the large eigenvalues is still resolved in time intervals independent of $\epsilon$, and thus their computational complexity is still formally $\mathcal{O}\left(\epsilon^{-1}\right)$. We point out, however, that some of these methods could be adopted as micro-solvers under our proposed methods in appropriate contexts.

Recently, Iserles [15] analyzed the accumulation of global error and showed that a class of "modified Magnus methods" permits larger time steps and exhibits good long-term behavior for a class of highly oscillatory linear systems. There are also methods that prepare the initial data such that the effect of the stiffness will not appear in the solutions. Kreiss wrote a series of papers on stiff ODE systems; see [18, 19, 20]. These results can be summarized by the "bounded derivative principle". However, in many common situations, initial values are given and the bounded derivative principle cannot be applied.

In the context of solving systems with the unique invariant manifold, the HMM schemes prepare the initial data naturally as a passive calculation to the effective force estimation. It is also clear that the method of [11] can also be interpreted as a scheme that benefits from the bounded derivative principle by following the transients to project onto the slow manifold.

Finally, for certain classes of singularly perturbed systems, hybrid analytic/ numerical methods [30, 31] and 33] have been proposed using related techniques. In these methods, an asymptotic expansion in the orders of $\epsilon$ is generated so that each coefficient is the sum of a slowly-varying function and an oscillating one. The oscillating part is solved analytically for efficiency.

We point out that our averaging approach is not directly applicable to stochastic equations. Instead, multiple realizations might be used to speed up the convergence. We refer the readers to the work of [8, 23] and [7. There is also a related paper on the analysis of heterogeneous multiscale methods for ODEs by Weinan E [5]. We should also point out here that one can generalize the HMM construct to systems with more than one scale. However, we will restrict our attention to systems with two scales in this paper.

The rest of this paper is structured as follows. In Section 2, we describe the framework of our proposed methods, including the theory related to using compactly supported kernels for effective force estimation. In Section 3, we start with a stability analysis of the simplest HMM ODE schemes for stiff dissipative and oscillatory problems. This is followed by a more detailed exposition of two major types of higher-order variants of the simple HMM ODE schemes, namely, the ones that are built from Runge-Kutta methods and those from linear multistep methods. 
Section 4 contains numerical results of some model problems. These numerical results confirm the theoretical results that we obtained in Sections 2 and 3. Finally, in the last section, we summarize this paper and also discuss some additional aspects of our methods.

\section{Approximation of effective force}

2.1. Estimation of the effective force. After Step 1(b), $u_{\epsilon, n}$ is a known function, and so we simplify our notation on the forces in this section by writing $f_{\epsilon}(t)$ instead of $f_{\epsilon}\left(u_{\epsilon, n}(t), t\right)$.

In our formulation, we need to estimate the effective force locally at a point using the microscale data (Step 1(b)-(d)). Motivated by the analytic averaging techniques, see e.g. [1, 2], we hypothesize that the effective force of a system of interest can be defined by

$$
\bar{f}(t)=\lim _{\delta \longrightarrow 0}\left[\lim _{\epsilon \longrightarrow 0} \frac{1}{\delta} \int_{t}^{t+\delta} f_{\epsilon}(\tau) d \tau\right] .
$$

We assume that $\bar{f}$ is slowly varying in the sense that

$$
\left|\frac{d^{p}}{d t^{p}} \bar{f}(t)\right| \leq C \text { for } 0 \leq p \leq s
$$

for some constant $C$ independent of $\epsilon$. Our goal in this section is to show that time averaging using a kernel $K_{\eta}^{p, q}$, defined below, with $\eta=\eta(\epsilon) \longrightarrow 0$ as $\epsilon \longrightarrow 0$ converges to $\bar{f}$ :

$$
K_{\eta}^{p, q} * f_{\epsilon}=K_{\eta}^{p, q} *\left(\bar{f}+g_{\epsilon}(t)\right) \longrightarrow \bar{f} \text { as } \epsilon \longrightarrow 0 .
$$

In many situations, $f_{\epsilon}$ or $g_{\epsilon}$ assumes special forms such as $f_{\epsilon}(t)=f_{\epsilon}(t, t / \epsilon)$ that are periodic in the second variable. For example,

$$
\frac{d u_{\epsilon}}{d t}=f_{\epsilon}\left(u_{\epsilon}, t\right)=\frac{i}{\epsilon} \lambda u_{\epsilon}+\phi(t)
$$

has solution

$$
u_{\epsilon}(t)=e^{i \epsilon^{-1} \lambda t}\left(u_{0}+\int_{0}^{t} e^{-i \epsilon^{-1} \lambda s} \phi(s) d s\right) .
$$

The force $f_{\epsilon}(t)=f_{\epsilon}(u, t)=\frac{i}{\epsilon} \lambda u_{\epsilon}+\phi(t)$ is of the form $f_{\epsilon}(t, t / \epsilon)$. In this case, we define

$$
\bar{f}(t)=\int_{0}^{1} f(t, s) d s
$$

and

$$
g_{\epsilon}(t)=g\left(t, \frac{t}{\epsilon}\right)=f_{\epsilon}-\bar{f}(t) .
$$

In this part of the paper, we show (2.1) in the cases that $g_{\epsilon}$ vanishes exponentially or oscillates at frequencies proportional to $\epsilon^{-1}$.

We will use $\mathbb{K}^{p, q}$ to denote the kernel space discussed in this paper. $K \in \mathbb{K}^{p, q}(I)$ if $K \in C_{c}^{q}(\mathbb{R})$ with $\operatorname{supp}(K)=I$, and

$$
\int_{\mathbb{R}} K(t) t^{r} d t= \begin{cases}1, & r=0 \\ 0, & 1 \leq r \leq p .\end{cases}
$$


Furthermore, we will use $K_{\eta}(t)$ to denote the scaling of $K$ :

$$
K_{\eta}(t):=\frac{1}{\eta} K\left(\frac{t}{\eta}\right)
$$

For convenience, we will also use $K^{p, q}$ to denote a function in $\mathbb{K}^{p, q}(I)$.

In the following proofs, we assume that $\operatorname{supp}(K)=[-1,1]$. However, it is clear that the proofs are also valid for the other case.

Notation 2.1. We use the notation $f^{[s]}(t)$ for the $s^{t h}$ order integral of $f$ from 0 with a constant that is specified from case to case:

$$
g_{\epsilon}^{[s]}(t)=\int_{0}^{t} g_{\epsilon}^{[s-1]}(y) d y+C_{s} .
$$

Also, we will use $f^{(s)}(t)$ to denote $d^{s} f / d t^{s}$. In particular, $f^{[0]}(t)=f^{(0)}(t)=f(t)$.

The following well-known results show that with suitable kernels, the $K_{\eta} * \bar{f}$ approximate $\bar{f}$ well.

Lemma 2.2. The following are well-known results.

(1) For any $f \in W^{\infty, p}(\mathbb{R}), K \in \mathbb{K}^{s, q}$,

$$
\left|K_{\eta} * f(t)-f(t)\right| \leq C_{f} \eta^{\max (p, s+1)} .
$$

(2) Let $g \in C(\mathbb{R})$. Then for any $K \in \mathbb{K}^{p, q}, \epsilon>0$,

$$
\left|K_{\eta} * g(\cdot / \epsilon)\right| \leq\left(\frac{\epsilon}{\eta}\right)^{q}\left\|g^{[q]}\right\|_{\infty}\|K\|_{W^{1, q}} .
$$

(3) If $\left|g\left(t_{0}, t\right)\right| \leq g_{0} \exp \left(t_{0}-t\right)$ for $t_{0} \leq t \leq T$, then for any $K \in \mathbb{K}^{p, q}([-1,0]) \cap$ $C_{c}^{q}\left(\left[-1,-\zeta_{0}\right]\right)$ for some $0 \leq \zeta_{0} \leq 1$, then

$$
\left|K_{\eta} * g\left(t_{0}, \cdot / \epsilon\right)\left(t_{0}\right)\right| \leq C_{0}\left(\frac{\epsilon}{\eta}\right)^{q} e^{-\zeta_{0} / \epsilon}|| K \|_{W^{1, q}} .
$$

(4) If $g \in C(\mathbb{R})$ such that $g(t+\alpha)=g(t), \int_{0}^{\alpha} g(t) d t=0$, for some $\alpha>0$ and $|g(t)| \leq C$ for all $0<\epsilon<\epsilon_{0}$, then for any $K \in \mathbb{K}^{p, q}$ and $\epsilon>0$,

$$
\left|K_{\eta} * g(\cdot / \epsilon)(t)\right| \leq \hat{C} \cdot \alpha^{q}\left(\frac{\epsilon}{\eta}\right)^{q}\|K\|_{W^{1, q}} .
$$

Proof. We omit the first two well known facts.

(3) Define $g^{[j]}\left(t_{0}, t\right)=\int_{t_{0}}^{t} g^{[j-1]}\left(t_{0}, \tau\right) d \tau$ for $j=1,2,3, \ldots$ Then $\left|g\left(t_{0}, t / \epsilon\right)^{[j]}\right| \leq$ $g_{0} \epsilon^{j} \exp \left(t_{0}-t / \epsilon\right)$ for $t_{0} \leq s / \epsilon \leq T$. Hence, by (2), we have the desired estimate.

(4) Define $g^{[j]}(t)=\int_{0}^{t} g^{[j-1]}(s) d s-\alpha^{-1} \int_{0}^{\alpha} \int_{0}^{t} g^{[j-1]}(s) d s d t$, for $j=1,2,3, \ldots$ We have that $g^{[j]}(t+a)-g^{[j]}(t)=0$ is a periodic function with zero average and since

$$
\begin{aligned}
\left|g^{[1]}(t)\right| & =\left|\alpha^{-1} \int_{0}^{\alpha} \int_{0}^{t} g(s) d s d \bar{t}-\alpha^{-1} \int_{0}^{\alpha} \int_{0}^{\bar{t}} g(s) d s d \bar{t}\right| \\
& =\left|\alpha^{-1} \int_{0}^{\alpha} \int_{\bar{t}}^{t} g(s) d s d t\right| \\
& \leq \alpha C,
\end{aligned}
$$

by induction, we have $\left|g^{[j]}(t)\right| \leq \alpha^{j} C$. Hence, induction by parts yields the desired estimate. 
We first investigate the dissipative case for which we average with a nonsymmetric kernel in $\mathbb{K}^{p, q}([-1,0])$. The following theorem shows that this approach lessens the stiffness.

Theorem 2.3. Let $f_{\epsilon}(t)=\bar{f}(t)+g\left(t_{0}, t / \epsilon\right)$, where $\left|g\left(t_{0}, s\right)\right| \leq C_{0} \exp \left(t_{0}-s\right)$ for $0 \leq t \leq T$. For any $K \in \mathbb{K}^{p, q}([-1,0]) \cap C_{c}^{q}\left(\left[-1,-\zeta_{0}\right]\right)$ for some $0 \leq \zeta_{0} \leq 1$, then there exist constants $C_{1}$ and $C_{2}$, independent of $\epsilon$ and $\eta$, such that

$$
E=\left|K_{\eta} * f_{\epsilon}(t)-\bar{f}(t)\right| \leq C_{1} \eta^{p}+C_{2}\left(\frac{\epsilon}{\eta}\right)^{q} e^{-\zeta_{0} \eta / \epsilon}\|K\|_{W^{1, q}} .
$$

In the rest of this section, we concentrate on the oscillatory case.

Lemma 2.4. If $g(t, s)=a(t) b(s)$, where $b(s+\alpha)=b(s), \int_{s}^{s+\alpha} b(\tau) d \tau=0$, for some $\alpha>0$, and $a \in C^{q}(\mathbb{R})$ and $\left\|a^{(q)}\right\|_{\infty} \leq M$, then for any $K \in \mathbb{K}^{p, q}$,

$$
\left|K_{\eta} * g(\cdot, \cdot / \epsilon)(t)\right| \leq C \alpha^{q}\left(\frac{\epsilon}{\eta}\right)^{q} \max _{0 \leq r \leq q}\left\|a^{(r)}\right\|_{\infty}\|K\|_{W^{1, q}}
$$

Proof. Let $\tilde{K}_{\eta}(t, s)=\eta^{-1} K((t-s) / \eta) a(s)$. Then $\tilde{K}(\cdot, s) \in C_{c}^{q}(\mathbb{R})$ and has the same support as $K$. We apply integration by parts by treating $\tilde{K}$ as our new kernel:

$$
\left|K_{\eta} * g(\cdot, \cdot / \epsilon)(t)\right|=\left|\int \tilde{K}_{\eta}(t, s) b(s / \epsilon) d s\right| \leq \epsilon^{q} \int\left|\tilde{K}_{\eta}^{(q)}(t, s) b^{[q]}(s / \epsilon)\right| d s .
$$

Here, $b^{[q]}$ are constructed in the same way as $g^{[q]}$ in the proof for item (4) in Lemma 2.2. Assuming that $\eta<1$,

$$
\begin{aligned}
\int\left|\frac{\partial^{q}}{\partial y^{q}} \tilde{K}_{\eta}(t, s)\right| d s & =\int\left|\sum_{r=0}^{q}\left(\begin{array}{c}
q \\
r
\end{array}\right)\left(-\frac{1}{\eta}\right)^{r} \frac{1}{\eta} K^{(r)}\left(\frac{t-s}{\eta}\right) a^{(q-r)}(s)\right| d s \\
& \leq \frac{\tilde{C}}{\eta^{q}}\|a\|_{W^{\infty, q}}\|K\|_{W^{1, q}} .
\end{aligned}
$$

$\left\|b^{[q]}\right\|_{\infty}$ is bounded the same way as in Lemma 2.2. The estimate follows.

Theorem 2.5. Let $f(t, s)$ be a 1-periodic function in the second variable whose derivative $\partial^{r} f(t, s) / \partial t^{r}$ is continuous and bounded by $C_{f}$ for $r=0, \ldots, \sigma+1$, and $\sigma>0$. Denote $f_{\epsilon}(t)=f(t, t / \epsilon)$ and define

$$
\bar{f}(t)=\int_{0}^{1} f(t, s) d s
$$

and

Then for any $K \in \mathbb{K}^{p, q}$,

$$
g\left(t, \frac{t}{\epsilon}\right)=f_{\epsilon}-\bar{f}(t)
$$

$$
K_{\eta} *\left(f_{\epsilon}-\bar{f}\right)(t)=C_{1}\|K\|_{W^{1, q}}\left(\frac{\epsilon}{\eta}\right)^{q}+C_{2} \eta^{\sigma+1} .
$$

Proof. Note that $\int_{0}^{1} g(t, s) d s=\int_{0}^{1}(f(t, s)-\bar{f}(t)) d s=0$ and $\frac{\partial^{k}}{\partial t^{k}} g(t, s+1)=$ $\frac{\partial^{k}}{\partial t^{k}} g(t, s)$ for $k=0,1, \ldots, \sigma$. Define, for each $k=1,2, \ldots, \sigma$,

$$
\frac{\partial^{k}}{\partial t^{k}} g^{[j]}(t, s)=\int_{0}^{s} \frac{\partial^{k}}{\partial t^{k}} g^{[j-1]}(t, \tilde{s}) d \tilde{s}-\int_{0}^{1} \int_{0}^{\tau} \frac{\partial^{k}}{\partial t^{k}} g^{[j-1]}(t, s) d s d \tau
$$

for $j=1,2,3, \ldots$ Then $\left|\frac{\partial^{k}}{\partial t^{k}} g^{[j]}(t, s)\right| \leq C_{f}$ due to the periodicity of $\frac{\partial^{k}}{\partial t^{k}} g(t, s)$ in $s$. 
Grouping the Taylor expansion of $g(t, s), K_{\eta} * g(\cdot, \dot{\bar{\epsilon}})(t)$ can be written as the sum of $I_{1}+I_{2}$, where

$$
I_{1}=\sum_{k=0}^{\sigma} \frac{1}{\eta} \int_{t-\eta}^{t+\eta} K\left(\frac{t-s}{\eta}\right) \frac{(t-s)^{k}}{k !} \frac{\partial^{k}}{\partial t^{k}} g\left(t, \frac{s}{\epsilon}\right) d s
$$

and

$$
I_{2}=\frac{1}{\eta} \int_{t-\eta}^{t+\eta} \int_{s}^{t} K\left(\frac{t-s}{\eta}\right) \frac{(t-\xi)^{\sigma}}{\sigma !} \frac{\partial^{\sigma+1}}{\partial t^{\sigma+1}} g\left(\xi, \frac{s}{\epsilon}\right) d \xi d s .
$$

Then, by Lemma 2.4

$$
\left|I_{1}\right| \leq \tilde{C}_{1}\left(\frac{\epsilon}{\eta}\right)^{q} \max _{0 \leq k \leq \sigma} \sup _{t} \sup _{s}\left|\frac{\partial^{k}}{\partial t^{k}} g^{[q]}(t, s)\right|\|K\|_{W^{1, q}} \sum_{k=1}^{\sigma} \frac{\eta^{k}}{k !}
$$

and

$$
\left|I_{2}\right| \leq \tilde{C}_{2} \frac{(\eta)^{\sigma+1}}{(\sigma+1) !} \sup _{t} \sup _{s}\left|\frac{\partial^{\sigma+1}}{\partial t^{\sigma+1}} g(t, s)\right|\|K\|_{L^{1}} .
$$

Hence, we can find $C_{1}$ and $C_{2}$ such that

$$
K_{\eta} *\left(f_{\epsilon}-\bar{f}\right)(t)=C_{1}\|K\|_{W^{1, q}}\left(\frac{\epsilon}{\eta}\right)^{q}+C_{2} \eta^{\sigma+1} .
$$

Remark 2.6. If $f_{\epsilon}(t)=f(t, a(t) / \epsilon), f(t, s)$ periodic in $s$, and $0<C_{1} \leq\left|a^{\prime}(t)\right| \leq C_{2}$, we can obtain similar bounds by the above procedures.

Hence, we have the estimate for the oscillatory case:

Theorem 2.7. Let $f_{\epsilon}(t)=f(t, t / \epsilon)$, where $f(t, s)$ is 1-periodic in the second variable and $\partial^{r} f(t, s) / \partial t^{r}$ is continuous for $r=0, \ldots, p-1$. For any $K \in \mathbb{K}^{p, q}$ there exists constants $C_{1}$ and $C_{2}$, independent of $\epsilon$ and $\eta$, such that

$$
E=\left|K_{\eta} * f_{\epsilon}(t)-\bar{f}(t)\right| \leq C_{1} \eta^{p}+C_{2}\left(\frac{\epsilon}{\eta}\right)^{q} .
$$

Furthermore, the error is minimized if $\eta$ is chosen to scale with $\epsilon^{q /(p+q)}$.

In the scheme illustrated by Figure 1.1, a nonsymmetric kernel should be used to evaluate $f$ at the beginning of each microscale evolution. The subfigures (a) and (b) in Figure 2.1 show the graph of such a kernel. On the contrary, in the schemes in Figure 1.2, one can use a symmetric kernel to estimate $f$ at the center of the time interval of each microscale evolution. A typical symmetric kernel is shown in the subfigure (c) of Figure 2.1. Most of the numerical examples of this paper are obtained from using the exponential kernel $K^{\exp } \in \mathbb{K}^{1, \infty}([-1,1])$ :

$$
K^{\exp }(t)=C_{0} \chi_{[-1,1]}(t) \exp \left(5 /\left(t^{2}-1\right)\right)
$$




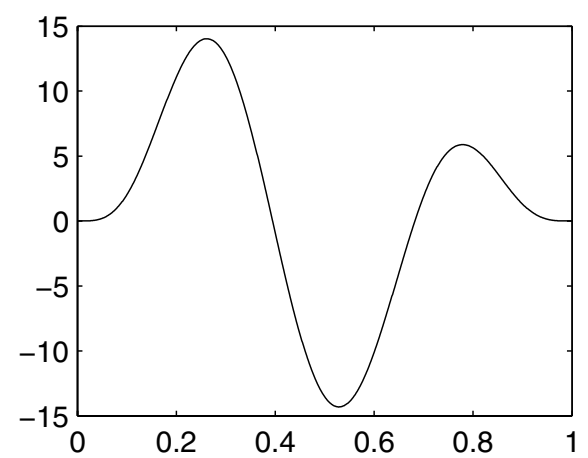

(a)

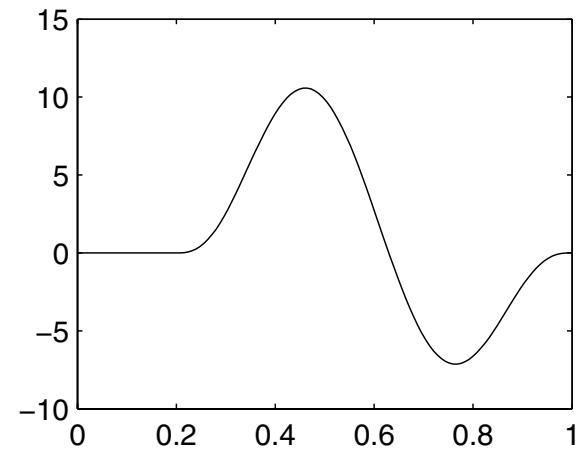

(b)

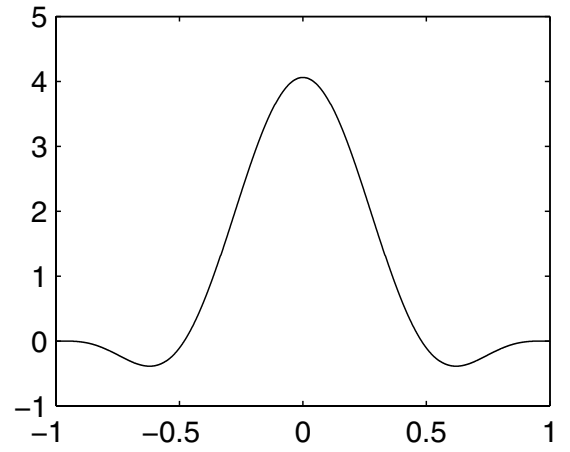

(c)

Figure 2.1. (a) An example of an asymmetric kernel in $\mathbb{K}^{2,3}([-1,0])$. (b) A shifted kernel in $\mathbb{K}^{1,2}([-1,0])$ for dissipative systems. (c) An example of a symmetric kernel in $\mathbb{K}^{2,3}([-1,1])$.

with $C_{0}$ adjusted so that $\left\|K^{\exp }\right\|_{L^{1}(\mathbb{R})}=1$. Another commonly used kernel is

$$
K^{\cos }(t)=\frac{1}{2} \chi_{[-1,1]}(t)(1+\cos (\pi t)) .
$$

Figure 2.2 demonstrates the residuals of using $K_{0.1}^{\exp }$ and $K_{0.1}^{\cos }$ for averaging $y_{n}=$ $\cos \left(t_{n} / \epsilon\right), t_{n}=n * 2 \pi \epsilon / 11$. 


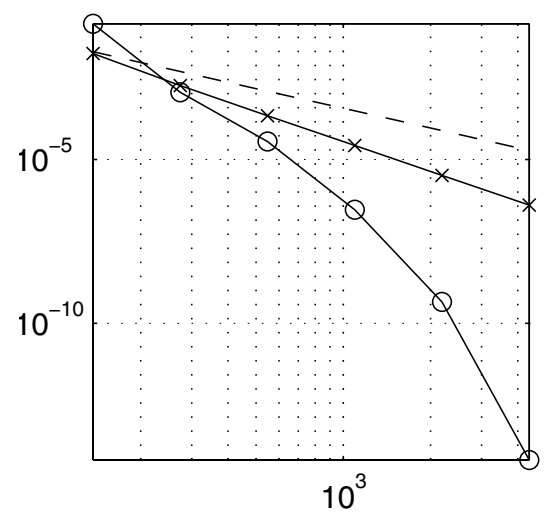

Figure 2.2. Averaging $y_{n}=\cos \left(t_{n} / \epsilon\right), t_{n}=n * 2 \pi \epsilon / 11,0 \leq t_{n} \leq$ $\eta$. The horizontal axis is $1 / \epsilon$. The circled data points are averages obtained by $K_{0.1}^{\exp }$ with respect to different $\epsilon$ 's, and crosses represent data points obtained by using $K_{0.1}^{\text {cos }}$. The line without crosses or circles is the graph of $C \epsilon$ for some $C$, adjusted so that the line lies in its current location. The dashed line indicates second-order accuracy with respect to $\eta$.

\section{Analysis of the HMM SChemes}

In the previous section, we presented the building blocks of an HMM scheme and the theory of averaging using a special class of kernels. In this section, we show various properties of the HMM schemes, including convergence.

We have to define what we mean by convergence such that it makes sense for very stiff problems $(\epsilon \ll H)$. For a given $\epsilon>0$, all well-known methods will converge as the stepsize $H \rightarrow 0$ and there is no difference between stiff and nonstiff problems. Therefore, we define the limiting error $E$ :

$$
E=\max _{n}\left(\lim _{H \rightarrow 0}\left(\sup _{0<\epsilon<\epsilon_{0}(H)}\left|U\left(t_{n}\right)-U^{n}\right|\right)\right),
$$

with $t_{n}=n H, \epsilon_{0}(H) / H \rightarrow 0$ as $H \rightarrow 0$.

We commence with linear systems. We remind the readers that in many of the dissipative cases analyzed below, since the reconstruction operator $R$ and the compression operator $Q$ are the identities, there is no difference in $u_{\epsilon}$ and $U$ after the transient. Thus we will use $u_{\epsilon, n}$ to denote the approximation of $u_{\epsilon}$ (and hence $U$ ) at $t_{n}$. So in the following presentation, our notation will reflect this fact.

3.1. Linear systems. We first discuss the properties of the HMM schemes for linear constant coefficient equations; i.e., $A(t)$ is a constant matrix $A$ in (1.2).

The HMM operations for force evaluation in combination with the Runge-Kutta schemes or Linear Multisteps schemes commute with the diagonalizer $S$ and $S^{-1}$. 
Take an HMM-LMM-rk scheme for example, and for simplicity, assume that $\phi(t)=$ 0 in (1.2). The force evaluation at $t_{n}$ is

$$
\begin{aligned}
\tilde{f}\left(U^{n}, t_{n}\right) & =\sum_{j=0}^{2 m} h K\left(t_{n}, t_{j}\right)\left(I+p\left(h A_{\epsilon}\right)\right)^{j} u_{\epsilon, n} \\
& =S \sum_{j=0}^{2 m} h K\left(t_{n}, t_{j}\right)\left(I+p\left(h \Lambda_{\epsilon}\right)\right)^{j} S^{-1} u_{\epsilon, n} .
\end{aligned}
$$

With $w^{n}=S^{-1} u_{\epsilon, n}$, and $W^{n}$ representing the macro variable (as $U$ to $u_{\epsilon, n}$ ), the original system is equivalent to $w^{\prime}=\Lambda_{\epsilon} w=g_{\epsilon}(w)$, and the force evaluation can be rewritten as

$$
\tilde{f}\left(U^{n}, t_{n}\right)=S \sum_{j=0}^{2 m} h K\left(t_{n}, t_{j}\right)\left(I+p\left(h \Lambda_{\epsilon}\right)\right)^{j} w^{n}=S \tilde{g}\left(W^{n}, t_{n}\right) .
$$

Therefore, the HMM-LMM-rk scheme takes the form

$$
\sum_{j=0}^{k} a_{k} S W^{n-j}=H \sum_{l=0}^{k} b_{l} S \tilde{g}\left(W^{n-l}\right)\left(W^{n-l}\right) .
$$

With the assumption that $S$ is independent of $\epsilon$, the HMM solution for $W$ is then equivalent to $S^{-1} U$. Hence, it suffices to investigate the stability and convergence issues by looking at the fully diagonalized system

$$
\frac{d u_{\epsilon}}{d t}=\lambda_{\epsilon}^{(j)} u_{\epsilon}
$$

for every $\lambda_{\epsilon}^{(j)}$ is an eigenvalue of $A_{\epsilon}$.

We present the stability and convergence properties of some basic HMM schemes built upon Runge-Kutta or Linear Multistep Methods.

3.1.1. Dissipative systems. We consider the case where $\lambda_{\epsilon}^{(j)}<0$ and the stiff components decay with exponential factors in the $\epsilon$ scale; i.e., the solution is attracted to the invariant manifold in the $\epsilon$ time scale. The bounded derivative principle [20] applies to this case, and one can prepare the initial data so that the fast scale is never excited. To prepare the initial data, one can simply evolve the solution $u_{\epsilon}$ for a small time duration $\eta$ so that $u_{\epsilon}$ is sufficiently close to or on the invariant manifold. Thus for the one-step HMM method, one can set $t_{*}=\eta$ in Step 1 of the Algorithm presented in Section 2, and simply take the values of $\bar{f}\left(t_{n}+\eta\right)=f_{\epsilon}\left(u_{\epsilon}\left(t_{n}+\eta\right), t_{n}+\eta\right)$; i.e., the kernel $K$ is the Dirac- $\delta$ function concentrated at $\eta$. Notice that one-step methods are particularly convenient for these problems, since $U\left(t_{n}+\eta\right)=u_{\epsilon}\left(t_{n}+\eta\right)$, and the solution $u_{\epsilon}$ is actually also obtained at $t_{k}^{l}=t_{0}+k(\eta+H)+l h, 0 \leq l \leq \mu$, with $\eta=m h$. Hence, we will present the following theorems only in $u_{\epsilon}$ instead of switching between $U$ and $u_{\epsilon}$.

When a linear multistep method is adopted as the macro-solver, $u_{\epsilon}\left(t_{0}\right)$ cannot be used directly as the initial values. Instead, one should prepare the initial value by $Q u_{\epsilon}=\tilde{K} * u_{\epsilon}\left(t_{0}\right)$, with $\tilde{K} \in \mathbb{K}_{\eta}^{p, q}$ depicted in Figure 2.1(b).

The following theorem shows the convergence of one-step HMM-RK-rk methods in the original variable $u_{\epsilon}$. 
Theorem 3.1. Let $u_{\epsilon}(t)$ be the analytical solution for (1.2) with $A_{\epsilon}(t) \equiv A_{\epsilon}$, and let $u_{\epsilon, n}$ be the approximation at $t_{n}$ computed by an HMM-RK-rk scheme:

$$
u_{\epsilon, n+1}=\left(I+P\left(H A_{\epsilon}\right)\right)\left(I+P\left(h A_{\epsilon}\right)\right)^{m} u_{\epsilon, n}, \quad u_{\epsilon, 0}=u_{\epsilon}\left(t_{0}\right) .
$$

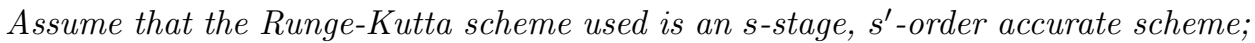
i.e., $P(z)$ is an $s$-degree polynomial in $z$.

Then for fixed $H$ and $\epsilon_{0}, \epsilon_{0}<H$, there is a constant $C_{\text {micro }}$, dependent on the micro-solver, and $n_{0}>0$ such that for $n \geq n_{0}$,

$$
\left|u_{\epsilon, n}-u_{\epsilon}\left(t_{n}\right)\right| \leq H^{s^{\prime}}
$$

if

(1) $\rho=|1+P(h / \epsilon)|<1$,

(2) $\eta=m h<H$,

$$
m \geq \frac{\mid \log \left(C_{\text {micro }}+C_{\text {micro }} \max _{j}\left|H \lambda_{\epsilon}^{(j)}\right|^{s}\right)}{|\log \rho|} \geq \frac{\log C_{\text {micro }}}{|\log \rho|}+s \frac{\max _{j}\left|\log H \lambda_{\epsilon}^{(j)}\right|}{|\log \rho|},
$$

$$
h^{s^{\prime}} \leq \frac{1}{C_{\text {micro }}} \frac{\epsilon^{s+s^{\prime}+1} H^{s^{\prime}+1} e^{n_{0}(H+\eta) / \epsilon}}{\eta\left(\epsilon^{s}+H^{s}\right)} .
$$

Proof. As we argued earlier, it suffices to show the convergence of the scheme on the fully decoupled equations. Therefore, we prove our theorem for the case:

$$
u_{\epsilon}^{\prime}=-\epsilon^{-1} u_{\epsilon}+\phi(t) .
$$

Define $\left(u_{\epsilon, n}\right)^{l}=\left(I+P\left(h A_{\epsilon}\right)\right)^{l} u_{\epsilon, n}$, and the error $E_{l}^{n}=u_{\epsilon}\left(t_{n}+l h\right)-\left(u_{\epsilon, n}\right)^{l}$, where $t_{n}=t_{0}+n(H+\eta)$, and $E^{n}=E_{0}^{n}=u_{\epsilon}\left(t_{n}\right)-u_{\epsilon, n}$. Then

$$
E_{l+1}^{n}=(I+P(-h / \epsilon)) E_{l}^{n}+r_{l}^{n},
$$

where $r_{l}^{n}$ is the local truncation error of the scheme at $t_{n}^{l}$. Let $Q_{h}=(I+P(-h / \epsilon))$ and $Q_{H}=(I+P(-H / \epsilon))$. Then we have

$$
E_{m}^{n}=\left(Q_{h}\right)^{m} E_{0}^{n}+\sum_{l=0}^{m-1}\left(Q_{h}\right)^{m-l-1} r_{l}^{n}
$$

and

$$
E^{n+1}=\left(Q_{H}\right)\left(Q_{h}\right)^{m} E^{n}+\mathcal{E}_{H M M}^{n}+R^{n},
$$

where $\mathcal{E}_{H M M}^{n}=Q_{H} \sum_{l=0}^{m-1}\left(Q_{h}\right)^{m-l-1} r_{l}^{n}$ can be considered as the local error committed by our HMM scheme at each macrostep, and $R^{n}$ is the local truncation error for the step from $t_{n}^{m}$ to $t_{n}^{m}+H$. Furthermore, let $Q_{H M M}=\left(Q_{H}\right)\left(Q_{h}\right)^{m}$ and assume $E^{0}=0$. Then we have

$$
E^{n+1}=\sum_{j=0}^{n} Q_{H M M}^{n-j}\left(\mathcal{E}_{H M M}^{j}+R^{j}\right) .
$$


The local truncation errors are bounded by $\left|r_{l}^{n}\right| \leq C_{r} \cdot(h / \epsilon)^{s^{\prime}+1} \exp (-(n H+n \eta+$ $l h) / \epsilon),\left|R^{n}\right| \leq C_{R}(H / \epsilon)^{s^{\prime}+1} \exp (-(n H+n \eta) / \epsilon)$, and

$$
\begin{aligned}
\left|\mathcal{E}_{H M M}^{n}\right| & \leq\left\|Q_{H}\right\| \sum_{l=0}^{m-1}\left\|Q_{h}^{m-l-1}\right\|\left|r_{l}^{n}\right| \\
& \leq C_{r} \frac{h^{s^{\prime}+1}}{\epsilon^{s^{\prime}+1}} e^{-n(H+\eta) / \epsilon}\left\|Q_{H}\right\| \sum_{l=0}^{m-1}\left\|Q_{h}\right\|^{m-l-1} e^{-l h / \epsilon} .
\end{aligned}
$$

We would like to show that when $h, \eta$, and $H$ satisfy certain conditions, $\left\|E^{n+1}\right\| \leq$ $H^{s^{\prime}}$; i.e., we need to bound

$$
\sum_{j=0}^{n}\left\|Q_{H M M}\right\|^{n-j}\left(\left|\mathcal{E}_{H M M}^{j}\right|+\left|R^{j}\right|\right) .
$$

It suffices to require that $\left\|Q_{H M M}\right\|^{k} \leq 1,\left|\mathcal{E}_{H M M}^{k}\right| \leq H^{s^{\prime}+1}$, and $\left|R^{k}\right| \leq H^{s^{\prime}+1}$ for $0 \leq k \leq n$. However, we require, additionally, the stability conditions for the micro-solver:

and the HMM scheme:

$$
\rho=|1+P(-h / \epsilon)|<1,
$$

$$
|I+P(-H / \epsilon)||I+P(-h / \epsilon)|^{m} \leq 1
$$

A sufficient condition for $\left|R^{k}\right| \leq H^{s^{\prime}+1}$ is

$$
n(H+\eta) \geq \max \left(\log \frac{1}{C_{R}}, s^{\prime}+1\right) \epsilon(1+|\log \epsilon|)=n_{0} .
$$

For an $s$-stage Runge-Kutta method, $P$ is a polynomial of degree $s$. There is a positive constant $C_{\text {micro }}$ depending on $P$ such that $|1+P(-H / \epsilon)| \leq$ $C_{m i c r o}\left(1+|H / \epsilon|^{s}\right)$, and the condition on the number of microsteps needed is estimated by

$$
m \geq \frac{\mid \log \left(C_{\text {micro }}+C_{\text {micro }}|H / \epsilon|^{s}\right)}{|\log \rho|} \geq \frac{\log C_{\text {micro }}}{|\log \rho|}+s \frac{|\log H / \epsilon|}{|\log \rho|} .
$$

$\left|\mathcal{E}_{H M M}^{k}\right| \leq H^{s^{\prime}+1}$ implies

$$
|1+P(-H / \epsilon)| \frac{h^{s^{\prime}} \eta}{\epsilon^{s^{\prime}+1}} e^{-n(H+\eta) / \epsilon} \leq H^{s^{\prime}+1},
$$

leading to

$$
h^{s^{\prime}} \leq \frac{1}{C_{\text {micro }}} \frac{\epsilon^{s+s^{\prime}+1} H^{s^{\prime}+1} e^{n(H+\eta) / \epsilon}}{\eta\left(\epsilon^{s}+H^{s}\right)} .
$$

We now turn to HMM-multistep schemes. Again, we consider the model linear system (1.2). Since effective force estimation, i.e. Step 1, commutes with matrix multiplications, we may simply consider the scalar case $f_{\epsilon}\left(u_{\epsilon}\right)=\lambda_{\epsilon} u_{\epsilon}$. The effective force $\bar{f}\left(U^{n}\right)$ is estimated by a kernel using accurate microscale data obtained near $t_{n}:$

$$
\bar{f}\left(U^{n}\right)=\lambda h \Sigma_{k} K_{\eta}\left(t_{n}-t_{k}\right)\left(\left(u_{\epsilon, n}\right)^{k}+\mathcal{O}\left(\left|\lambda_{\epsilon} h\right|^{p^{\prime}}\right) .\right.
$$

In fact, $\left(u_{\epsilon, n}\right)^{k}=U^{n} \exp \left(\lambda_{\epsilon} \cdot k h\right)$, and

$$
\begin{aligned}
\bar{f}\left(U^{n}\right) & =\lambda \Sigma_{k} h K_{\eta}\left(t_{n}-t_{n}^{k}\right)\left(e^{\lambda_{\epsilon} k h} U^{n}+\mathcal{O}\left(\left|\lambda_{\epsilon} h\right|^{p^{\prime}}\right)\right. \\
& =\lambda U^{n} \mathcal{A}\left(K, \lambda_{\epsilon}, \eta, h\right)+\mathcal{O}\left(\left|\lambda_{\epsilon} h\right|^{\min \left(\alpha, p^{\prime}\right)}\right),
\end{aligned}
$$


where $\alpha$ depends on the quadrature, and $\mathcal{A}\left(K, \lambda_{\epsilon}, \eta, h\right)$ denotes the weighted average of $\exp \left(\lambda_{\epsilon} k h\right), 0 \leq k h \leq \eta$. Hence, Equation (1.5) becomes

$$
\begin{gathered}
a_{0} U^{n}+a_{1} U^{n-1}+\cdots+a_{k} U^{n-k}=\lambda H \mathcal{A}\left(K, \lambda_{\epsilon}, \eta, h\right)\left(b_{0} U^{n}+b_{1} U^{n-1}+\cdots\right. \\
\left.+b_{k} U^{n-k}+\mathcal{O}\left(\left|\lambda_{\epsilon} h\right|^{\min \left(\alpha, p^{\prime}\right)}\right)\right) .
\end{gathered}
$$

Therefore, for stability, we need the root conditions for

$$
\left(a_{0}-\lambda H \mathcal{A}\left(K, \lambda_{\epsilon}, \eta, h\right) b_{0}\right) z^{k}+\cdots+\left(a_{k}-\lambda H \mathcal{A}\left(K, \lambda_{\epsilon}, \eta, h\right) b_{k}\right)=0 ;
$$

i.e., $|z| \leq 1$ for a non-multiple root and $|z|<1$ for a multiple root. $\mathcal{A}\left(K, \lambda_{\epsilon}, \eta, h\right)$ can be estimated by the results in Section 2.1 .

3.1.2. Dissipative systems with variable coefficients. We consider variable coefficient systems (1.2) with $\phi(t)=0$.

Theorem 3.2. For fixed $H$ and $0<\epsilon<\epsilon_{0}<H$, the HMM-FE-fe scheme

$$
u_{\epsilon, n+1}=\left(I+H A_{\epsilon}\left(t_{n}+\eta\right)\right) \Pi_{j=0}^{m-1}\left(I+h A_{\epsilon}\left(t_{n}+j h\right)\right) u_{\epsilon, n}
$$

is stable if $h$ and $n$ satisfy

$$
C\left(\epsilon_{0}+\tilde{C} H\right) \cdot\left(1-\tilde{C} \frac{h}{\epsilon}\right)^{n} \cdot \frac{h^{n}}{\epsilon^{n+1}} \leq 1,
$$

for some constant $C$. Here $\lambda_{\epsilon}^{(j)}(t)$ are the eigenvalues of $A_{\epsilon}$ and satisfy

$$
\max _{j, t \in[0, T]}\left|\lambda_{\epsilon}^{(j)}(t)\right|<\tilde{C} \epsilon^{-1}
$$

Proof. To simplify our notation, we will use $A_{n, k}$ and $S_{n, k}$ to denote $A_{\epsilon}\left(t_{n}+k h\right)$ and $S\left(t_{n}+k h\right)$, and $A_{n, k}=S_{n, k} \Lambda_{n, k} S_{n, k}^{-1}$. For $k \leq m-1$,

$$
\begin{gathered}
\left(u_{\epsilon, n}\right)^{k+1}=S_{n, k}\left(I+h \Lambda_{n, k}\right) S_{n, k}^{-1} S_{n, k-1}\left(I+h \Lambda_{n, k-1}\right) S_{n, k-1}^{-1} \cdots \\
S_{n, 0}\left(I+h \Lambda_{n, 0}\right) S_{n, 0}^{-1}\left(u_{\epsilon, n}\right)^{0} .
\end{gathered}
$$

Hence,

$$
\left\|\left(u_{\epsilon, n}\right)^{k+1}\right\| \leq \Pi_{j=0}^{k}\left\|I+h \Lambda_{n, j}\right\| \cdot \Pi_{l=1}^{k}\left\|S_{n, l}^{-1} S_{n, l-1}\right\| \cdot\left(\left\|S_{k}\right\|\left\|S_{0}\right\|\right) .
$$

We know that the columns of $S_{n, j}$ consist of the right eigenvectors of $A_{n, j}$. Using the mean value theorem, we can write

$$
S_{n, j-1}=S_{n, j}-h B_{n, j} .
$$

By the perturbation theory of linear operators, see e.g. [16],

$$
B_{n, j}=\left(b_{l_{1} l_{2}}\right)=\left\{\begin{array}{l}
\tilde{a}_{l_{1} l_{2}} /\left(\lambda_{\epsilon}^{\left(l_{1}\right)}-\lambda_{\epsilon}^{\left(l_{2}\right)}\right), \text { if } l_{1} \neq l_{2}, \\
0, \text { if } l_{1}=l_{2},
\end{array}\right.
$$

where $\lambda_{\epsilon}^{(j)}$ are the eigenvalues of $A_{\epsilon}\left(t_{n}+j h\right)$. Under the hypotheses on the eigenvalues,

$$
B_{k}=\epsilon^{-1} \tilde{B}_{k}=\epsilon^{-1}\left(\begin{array}{ll}
\mathbb{B}_{11} & \mathbb{B}_{12} \\
\mathbb{B}_{21} & \mathbb{B}_{22}
\end{array}\right)
$$

and $\mathbb{B}_{22}$ is a $\left(d-k_{0}\right) \times\left(d-k_{0}\right)$ matrix of $\mathcal{O}(1)$, while $\mathbb{B}_{11}, \mathbb{B}_{12}$ and $\mathbb{B}_{21}$ are of $\mathcal{O}(\epsilon)$ and

$$
\begin{aligned}
\left\|S_{i}^{-1} S_{i-1}\right\| & =\left\|S_{i}^{-1}\left(S_{i}-\epsilon^{-1} h \tilde{B}_{i}\right)\right\| \\
& =\left\|I-\epsilon^{-1} h S_{i}^{-1} \tilde{B}_{i}\right\| \\
& \leq \text { Const } d h / \epsilon .
\end{aligned}
$$


Our chosen HMM-FE-fe scheme, in particular, can be written as

$$
y^{m+1}=\left(I+H A_{\epsilon}\left(t_{m}\right)\right) \Pi_{j=0}^{m-1}\left(I+h A_{\epsilon}\left(t_{m}+j h\right)\right) y_{0} .
$$

Now if $h \lambda(t)$ is always inside the regions of absolute stability for the forward Euler scheme, i.e., $h \max _{j, t \in[0, T]}\left|\lambda_{\epsilon}^{(j)}(t)\right|=\tilde{C} h / \epsilon<1$, then we have

$$
\begin{aligned}
\left\|y^{m+1}\right\| & \leq \text { Const }\left\|I+H A\left(t_{m+1}\right)\right\| \cdot(1-\tilde{C} h / \epsilon)^{m} \cdot \Pi_{i=0}^{m-1}\left\|S_{i}^{-1} S_{i-1}\right\| \\
& \leq \text { Const }(1+\tilde{C} H / \epsilon) \cdot(1-\tilde{C} h / \epsilon)^{m} \cdot \frac{h^{m}}{\epsilon^{m}} .
\end{aligned}
$$

Hence we have proved that HMM-FE-fe is stable if

$$
C\left(\epsilon_{0}+\tilde{C} H\right) \cdot(1-\tilde{C} h / \epsilon)^{m} \cdot \frac{h^{m}}{\epsilon^{m+1}} \leq 1
$$

for all $0<\epsilon \leq \epsilon_{0}$.

3.1.3. Oscillatory systems. Define $D^{+} \phi_{j}:=\left(\phi_{j+1}-\phi_{j}\right) / h, D^{-} \phi_{j}:=\left(\phi_{j}-\phi_{j-1}\right) / h$, and the discrete semi-norm for the grid function: $\left\|D^{-} \phi\right\|_{h}:=\sum_{j=0}^{m}\left|D^{-} \phi_{j}\right| h$. The following lemma shows the property of effective force estimation in a discrete setting (this is in parallel to Lemma 2.2).

Lemma 3.3. Let $g_{j}=(1+P(i h / \epsilon))^{j}=D^{+} G_{j}, G_{0}=0$ and $P(z)=\sum_{\nu=1}^{s} c_{\nu} z^{\nu}$. Let $w=\left\{w_{j} \in \mathbb{R}: w_{j}=0\right.$ for $j \leq 0$ or $\left.j \geq m\right\}$, and $C_{P}=\max _{1 \leq \nu \leq s}\left|c_{\nu}\right|$. Then

$$
\left|\sum_{j=0}^{m} w_{j} g_{j} h\right| \leq C_{p} \epsilon\left(1+\frac{h^{2}}{\epsilon^{2}}\right)^{m / 2}\left\|D^{-} w\right\|_{h} .
$$

In particular, if $w_{j}=K(-j h / \eta) / \eta$ corresponds to the grid values of the kernel $K_{\eta}$, then $\left|D^{-} w_{j}\right| \leq \eta^{-2}\left\|K^{\prime}\right\|_{\infty}$ where $\eta=m h$, and if $0<h / \epsilon<\rho_{0}<1$, then

$$
\left|\sum_{j=0}^{m} w_{j} g_{j} h\right| \leq C_{p} \frac{\epsilon}{\eta} \exp \left(\eta h \epsilon^{-2} / 2\right)\left\|K^{\prime}\right\|_{\infty}
$$

Proof. This is an application of summation by parts. We have $\sum_{j=0}^{m} w_{j} g_{j} h=$ $\sum_{j=0}^{m} w_{j}\left(G_{j+1}-G_{j}\right)=-\sum_{j=0}^{m} D^{-} w_{j} G_{j} h$. Since $G_{n+1}=G_{n}+h g_{n}=\left(G_{n-1}+\right.$ $\left.h g_{n-1}\right)+h g_{n} \cdots=\sum_{j=0}^{n} g_{j} h, G_{0}=0$

$$
\sum_{j=0}^{m} w_{j} g_{j} h=\sum_{j=0}^{m} D^{-} w_{j} G_{j} h=h^{2} \sum_{j=0}^{m} D^{-} w_{j} \frac{(1+P(i h / \epsilon))^{j+1}}{P(i h / \epsilon)} .
$$

With the hypothesis that $0<h / \epsilon<1$, there is a constant $C_{p}$ depending only on the polynomial $P$ such that

$$
|1+P(i h / \epsilon)|^{m} \leq C_{p}\left(1+h^{2} \epsilon^{-2}\right)^{m / 2} .
$$

Hence we have (3.2). In particular, if $w_{j}=K_{\eta}(-j h)$, then

$$
\left|D^{-} w_{j}\right|=\left|\frac{1}{\eta}\left(\frac{K\left(\frac{-j h}{\eta}\right)-K\left(\frac{-(j-1) h}{\eta}\right)}{h}\right)\right| \leq \eta^{-2}\left\|K^{\prime}\right\|_{\infty} .
$$

With $m h=\eta$,

$$
\left|\sum_{j=0}^{m} w_{j} g_{j} h\right| \leq C_{p} \frac{\epsilon}{\eta} \exp \left(\eta h \epsilon^{-2} / 2\right)\left\|K^{\prime}\right\|_{\infty}
$$


The following stability result is a direct consequence of the above lemma.

Theorem 3.4. Consider HMM-FE-rk methods with uniform macroscale step:

$$
U^{n+1}=\left(1+H \sum_{j=0}^{m} h \tilde{K}_{j}^{n}\left(1+P\left(\frac{i h}{\epsilon}\right)\right)^{j}\right) U^{n},
$$

where $\tilde{K}_{j}{ }^{n}=K\left(\frac{t_{n}-j h}{m h}\right)$ is the discretization of the kernel used, and $P(z)=$ $\sum_{\nu=1}^{s} c_{\nu} z^{\nu}$ is the polynomial corresponding to the Runge-Kutta scheme. Set $C_{R K}=$ $\max _{1 \leq \nu \leq s}\left|c_{\nu}\right|$. For fixed $H, \epsilon$, if $h$ is chosen such that $0<h / \epsilon<1$ and $\eta=m h$, $m>2$, then

$$
\left|U^{n+1}\right| \leq \exp \left(C_{R K} n H \frac{\epsilon}{\eta} e^{\eta h \epsilon^{-2} / 2}\right)\left|U^{0}\right| .
$$

The reason that we choose $m>2$ is to reflect our true algorithm. If we choose $m=1$, for example, the kernel used in our force evaluation is not resolved at all. In fact, the boundary conditions will result in the "summation" to be zero. In a later part of this section, we will analyze a model nonlinear problem. There, we will estimate how far the discrete operations approximate the continuum-level HMM operations such as convolution with a kernel.

We see that in this coarse estimate from the discrete scheme, the kernel estimation lessens the amplification factor from $\exp \left(H^{2} \epsilon^{-2} / 2\right)$ to $\exp \left(H \frac{\epsilon}{\eta} \exp \left(\eta h \epsilon^{-2} / 2\right)\right)$. (We omit the constants here for convenience.) We also remark that the same estimate holds (with a different constant, of course) if a variable time step HMM-FE-rk is used.

Theorem 3.5. HMM-LF-fe for $u^{\prime}=i \epsilon^{-1} u$ is

$$
U^{k+1}=U^{k-1}+2 H \sum_{j=0}^{m} h \tilde{K}_{j}\left(1+\frac{i h}{\epsilon}\right)^{j} U^{k} .
$$

Fix $q>0$, for any given $H, \epsilon, T=n H$, if $\eta=m h<C_{0} \epsilon^{1 / q}$, and $h<C_{1} \epsilon^{2-1 / q}$, then there is a constant $\tilde{C}$ independent of $\eta$ and $h$ such that

$$
\left|U^{k}\right| \leq \exp \left(\tilde{C} h \epsilon^{1-1 / q} T\right)
$$

for $1 \leq k \leq n$.

Proof. Direct calculations involving summation by parts show

$$
\begin{aligned}
U^{k+1} & =U^{k-1}+2 H h U^{k} \sum_{j=0}^{m} D^{-} \tilde{K}_{j} \frac{-(1+i h / \epsilon)^{j}}{i h / \epsilon} h \\
& =U^{k-1}+2 \epsilon h H U^{k} \sum_{j=0}^{m} D^{-} \tilde{K}_{j}\left(1+h^{2} \epsilon^{-2}\right)^{j / 2} e^{i j \theta},
\end{aligned}
$$

where $\left(1+h^{2} \epsilon^{-2}\right)^{1 / 2} \exp (i \theta)=\left(1+i h \epsilon^{-1}\right)$.

Let $\gamma_{m}=\sum_{j=0}^{m} D^{-} \tilde{K}_{j}\left(1+h^{2} \epsilon^{-2}\right)^{j / 2} \exp (i j \theta)$ and $\rho=\epsilon h H>0$. The characteristic roots for (3.4) are $z=\rho \gamma_{m} \pm\left(1+\rho^{2} \gamma_{m}^{2}\right)^{1 / 2}$ and $|z| \leq e^{2 \rho\left|\gamma_{m}\right|}$. From Lemma 3.3. we have $\left|\gamma_{m}\right| \leq\left\|K^{\prime}\right\|_{\infty}\left(1+h^{2} \epsilon^{-2}\right)^{m / 2} / \eta$. Therefore,

$$
|z|^{n} \leq \exp \left(2 \frac{\epsilon}{\eta} h\left(1+h^{2} \epsilon^{-2}\right)^{m / 2}\left\|K^{\prime}\right\|_{\infty} T\right)
$$


By the hypotheses, $h=C_{2} \epsilon^{2-1 / q},\left(1+h^{2} \epsilon^{-2}\right)^{m} \leq \exp \left(\eta h \epsilon^{-2}\right) \leq C_{4}$, and

$$
|z|^{n} \leq \exp \left(\tilde{C} h \epsilon^{1-1 / q} T\right) .
$$

Remark 3.6. The factor $\epsilon^{1-1 / q}$ in the estimate above shows the discrete averaging effect.

3.2. Nonlinear systems. Our main focus has been to discuss the HMM technique for stiff ODEs in general and to develop the convergence theory for linear problems. In this section, we shall briefly consider nonlinear problems and start with a simple class of systems. The purpose is to present examples for which it is easy to see how HMM methods converge but for which the standard ODE methods for stiff problems do not work. After the analysis, we shall also give numerical approximations of more general nonlinear systems.

3.2.1. Simple analytical examples. A simple class of systems has the form

$$
\left\{\begin{array}{l}
x^{\prime}=f_{\epsilon}^{I}(x, y, t)=i \epsilon^{-1} x+f^{I}(x, y, t), \\
y^{\prime}=f^{I I}(x, y, t),
\end{array}\right.
$$

with initial conditions $x(0)=x_{0}$ and $y(0)=y_{0}$, and Lipschitz continuous functions $f^{I}$ and $f^{I I}$.

Let us first give an example for which the implicit Euler method does not converge.

Example 3.7. Our first example to show that HMM schemes converge as $\epsilon \rightarrow 0$ is

$$
\left\{\begin{array}{l}
\dot{x}=i \epsilon^{-1} x \\
\dot{y}=|x|^{2}
\end{array},\left(\begin{array}{l}
x_{0} \\
y_{0}
\end{array}\right)=\left(\begin{array}{l}
1 \\
0
\end{array}\right),\right.
$$

whose solution is

$$
\left\{\begin{array}{l}
x=e^{i \epsilon^{-1} t} \\
y=t
\end{array}\right.
$$

In this case, we know that the effective force of the system is

$$
\bar{f}=\left(\begin{array}{l}
0 \\
1
\end{array}\right) .
$$

A typical HMM solution is

$$
\left\{\begin{array}{l}
X^{n+1}=C\left(\frac{\epsilon}{\eta}\right)^{q} e^{i \epsilon^{-1} \tau_{n}}, \\
Y^{n+1}=t_{n},
\end{array}\right.
$$

with $\tau_{n} \neq t_{n}$, and $t_{n}=n H$.

A typical stiff implicit method resembles the Implicit Euler scheme which will generate the solution

$$
\left\{\begin{array}{l}
x^{n+1}=(1-i H / \epsilon) x^{n}, \\
y^{n+1}=y^{n}+H\left|x^{n+1}\right|^{2} .
\end{array}\right.
$$

As $\epsilon \rightarrow 0$,

$$
x^{n} \rightarrow\left\{\begin{array}{l}
1, n=0, \\
0, n \geq 1,
\end{array}\right.
$$

and

$$
y^{n} \rightarrow 0 .
$$

$\left(x^{n}, y^{n}\right)$ is apparently a wrong solution. 
Notice that the trapezoidal rule works for the above example, but fails for slightly more nonlinear examples.

\section{Example 3.8.}

whose solution is

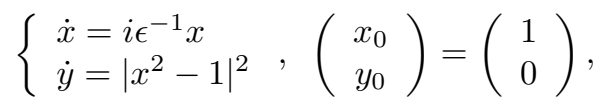

$$
\left\{\begin{array}{l}
x=e^{i \epsilon^{-1} t} \\
y=\frac{t}{2 \pi} \int_{0}^{2 \pi}(2-2 \cos \theta) d \theta .
\end{array}\right.
$$

However, the trapezoidal rule, in the limit of $\epsilon \rightarrow 0$, will give $x^{n}=1$ or -1 , and $y^{n}=0$.

We point out here a situation in which the simple HMM constructions, though stable, do not yield correct results.

Example 3.9. Consider (3.5). Let $u_{\epsilon}=(x, y)$ solve (3.5) with $f^{I} \equiv 1$ and $x(0)=$ $x_{0}$, and let $\hat{u}_{\epsilon}=(\hat{x}, \hat{y})$ solve (3.5) with $f^{I}(\hat{x}, \hat{y}, t)=\hat{x}$ and $\hat{x}(0)=x_{0} 1$ Note that under the settings on $f^{I}, x(t), \hat{x}(t)$ is independent of $y(t)$ and $\hat{y}(t)$.

One can write down the explicit forms of $u_{\epsilon}$ and $\hat{u}_{\epsilon}$ :

$$
\left\{\begin{array}{l}
x_{\epsilon}(t)=e^{i t / \epsilon}\left(x_{0}-\epsilon i\right)+\epsilon i, \\
\hat{x}_{\epsilon}(t)=x_{0} e^{t} e^{i t / \epsilon}
\end{array}\right.
$$

Therefore, we know that as $\epsilon \rightarrow 0$, both $x$ and $\tilde{x}$ converge weakly to 0 as well as $\phi_{1}$ and $\phi_{2}$. Let $U^{n}=\left(X^{n}, Y^{n}\right)$ and $\hat{U}^{n}=\left(\hat{X}^{n}, \hat{Y}^{n}\right)$ denote the corresponding macroscopic variables constructed by an HMM scheme. At a given time step, the direct averaging strategy (Step 1, with the identity operator as the reconstruction operator $R$ ) proposed earlier will approximate the weak limit of $\phi_{1}$ and $\phi_{2}$ and yield in the limit $X^{n}=\hat{X}^{n}=x_{0}$.

If the quantity $|x|$ is needed in the equation for $y$, e.g. if $f^{I I}(x, y, t)=|x|^{2}$, then the proposed HMM schemes do not converge, since Step 1 evaluates $\tilde{f}^{I I}\left(t_{n}\right)=$ $\left|\hat{X}^{n}\right| \equiv x_{0}$. However, $\bar{f}^{I I}=|\hat{x}|^{2}=\left|x_{0}\right|^{2} e^{2 t}$.

3.2.2. Model nonlinear oscillatory example. Let $u_{\epsilon}=\left(x_{\epsilon}, y_{\epsilon}\right)$ be the solution in $t \in\left[t_{0}, T_{1}\right]$ for

$$
\left\{\begin{array}{l}
\frac{d}{d t} x_{\epsilon}=\frac{i}{\epsilon} x_{\epsilon}+f^{I}\left(x_{\epsilon}, y_{\epsilon}\right), \\
\frac{d}{d t} y_{\epsilon}=f^{I I}\left(x_{\epsilon}, y_{\epsilon}\right),
\end{array}\right.
$$

with initial conditions $u_{\epsilon}\left(t_{0}\right)=u_{0}=\left(x_{0}, y_{0}\right)$. Let $x_{\epsilon}=e^{i \epsilon^{-1} t} w_{\epsilon}$. Then $\left(w_{\epsilon}, v_{\epsilon}\right)$ solves the following system:

$$
\left\{\begin{array}{l}
\frac{d}{d t} w_{\epsilon}=e^{-i \epsilon^{-1} t} f^{I}\left(e^{i \epsilon^{-1} t} w_{\epsilon}, v_{\epsilon}\right), \\
\frac{d}{d t} v_{\epsilon}=f^{I I}\left(e^{i \epsilon^{-1} t} w_{\epsilon}, v_{\epsilon}\right),
\end{array}\right.
$$

with initial conditions $w_{\epsilon}\left(t_{0}\right)=x_{0}$ and $v_{\epsilon}\left(t_{0}\right)=y_{0}$.

We define the averaged force

$$
\left\{\begin{array}{l}
\bar{f}^{I}(x, y)=\frac{1}{2 \pi} \int_{0}^{2 \pi} e^{-i \theta} f^{I}\left(e^{i \theta} x, y\right) d \theta \\
\bar{f}^{I I}(x, y)=\frac{1}{2 \pi} \int_{0}^{2 \pi} f^{I I}\left(e^{i \theta} x, y\right) d \theta
\end{array}\right.
$$

\footnotetext{
${ }^{1}$ This equation does not fall into the class of linear systems that we considered in the previous section.
} 
Denote $(\bar{w}, \bar{v})$ as the solution for

$$
\left\{\begin{array}{l}
\frac{d}{d t} \bar{w}=\bar{f}^{I}(\bar{w}, \bar{v}), \\
\frac{d}{d t} \bar{v}=\bar{g}^{I I}(\bar{w}, \bar{v}),
\end{array}\right.
$$

with initial conditions $\bar{w}\left(t_{0}\right)=u_{0}$ and $\bar{v}\left(t_{0}\right)=v_{0}$.

In the following discussion, we will assume that $f^{I}$ and $f^{I I}$ are smooth and bounded. However, most of the analysis carries over under a more relaxed condition that $f^{I}$ and $f^{I I}$ are Lipschitz continuous. We notice that $0<\epsilon \leq \epsilon_{0}$, there is a time $T_{1}$ and a constant $M$ such that the family of solutions $\left(w_{\epsilon}, v_{\epsilon}\right)$ to (3.7) exists and is bounded uniformly by $M$; i.e., $\left|w_{\epsilon}(t)\right|+\left|v_{\epsilon}(t)\right| \leq M$ for all $t \in\left[t_{0}, T_{1}\right]$ and for all $\epsilon \in\left(0, \epsilon_{0}\right]$; hence $\left|x_{\epsilon}(t)\right|+\left|y_{\epsilon}(t)\right| \leq M$. This can be established from the construction of solutions through Picard's iterations. Hence, given Lipschitz continuous functions $f$ and $g$, we also obtain a bound on the maximal values of $\left|f^{I}(x, y)\right|$ and $\left|f^{I I}(x, y)\right|$ as long as $|x|+|y| \leq M$. Hence, in the remainder of this section, we will just assume that $f^{I}$ and $f^{I I}$ are bounded functions.

Theorem 3.10. There exists $T^{\prime}$ and a constant $C$ such that for $t_{0} \leq t \leq T^{\prime}$, $\left|w_{\epsilon}-\bar{w}\right|+\left|v_{\epsilon}-\bar{v}\right| \leq C \epsilon$.

Proof. From basic existence theory, due to the smoothness assumption of $f^{I}$ and $f^{I I}$, and consequently $\bar{f}^{I}$ and $\bar{f}^{I I}, \exists T^{\prime}>t_{0}$ such that both $\left(w_{\epsilon}, v_{\epsilon}\right)$ and $(\bar{w}, \bar{v})$ exist in $\left[t_{0}, T^{\prime}\right]$.

We bound the differences $\bar{w}-w_{\epsilon}$ and $\bar{v}-v_{\epsilon}$ for $t \in\left[t_{0}, T^{\prime}\right]$. Let $\bar{L}$ be a positive constant such that $\left|\bar{f}^{I}\left(x_{1}, y_{1}\right)-\bar{f}^{I I}\left(x_{2}, y_{2}\right)\right| \leq \bar{L}\left(\left|x_{1}-x_{2}\right|+\left|y_{1}-y_{2}\right|\right)$ and $\left|\bar{f}^{I I}\left(x_{1}, y_{1}\right)-\bar{g}\left(x_{2}, y_{2}\right)\right| \leq \bar{L}\left(\left|x_{1}-x_{2}\right|+\left|y_{1}-y_{2}\right|\right)$. Then

$$
\begin{aligned}
\left|\bar{w}(t)-w_{\epsilon}(t)\right|= & \left|\int_{t_{0}}^{t} \bar{f}^{I}(\bar{w}(\tau), \bar{v}(\tau))-e^{-i \tau / \epsilon} f^{I}\left(e^{i \tau / \epsilon} w_{\epsilon}(\tau), v_{\epsilon}(\tau)\right) d \tau\right| \\
= & \left|\int_{t_{0}}^{t} \bar{f}^{I}(\bar{w}(\tau), \bar{v}(\tau))-\bar{f}^{I}\left(w_{\epsilon}(\tau), v_{\epsilon}(\tau)\right) d \tau\right| \\
& +\left|\int_{t 0}^{t} \bar{f}^{I}\left(w_{\epsilon}(\tau), v_{\epsilon}(\tau)\right)-e^{-i \tau / \epsilon} f^{I}\left(e^{i \tau / \epsilon} w_{\epsilon}(\tau), v_{\epsilon}(\tau)\right) d \tau\right| \\
\leq & \left(t-t_{0}\right) \bar{L} \sup _{\tau \in\left[t_{0}, \bar{T}\right]}\left(\left|\bar{w}(\tau)-w_{\epsilon}(\tau)\right|+\left|\bar{v}(\tau)-v_{\epsilon}(\tau)\right|\right)+I_{1},
\end{aligned}
$$

where $I_{1}=\left|\int_{t_{0}}^{t} \bar{f}^{I}\left(w_{\epsilon}(\tau), v_{\epsilon}(\tau)\right)-e^{-i \tau / \epsilon} f^{I}\left(e^{i \tau / \epsilon} w_{\epsilon}(\tau), v_{\epsilon}(\tau)\right) d \tau\right|$. We shall show that $0 \leq I_{1} \leq C \epsilon$ for some constant $C$.

Due to the periodicity of $\exp (i \tau / \epsilon)$, we can write down the above integrals as sums of averages:

$$
\begin{aligned}
& \int_{t_{0}}^{t} e^{-i \tau / \epsilon} f^{I}\left(e^{i \tau / \epsilon} w_{\epsilon}(\tau), v_{\epsilon}(\tau)\right) d \tau \\
& \quad=\sum_{i=0}^{n} \int_{t_{i}}^{t_{i}+2 \pi \epsilon} e^{-i t / \epsilon} f^{I}\left(e^{i t / \epsilon} w_{\epsilon}\left(t_{i}\right), v_{\epsilon}\left(t_{i}\right)\right) d t+R_{i}+I_{\epsilon}, \quad 0 \leq t-t_{n} \leq 2 \pi \epsilon,
\end{aligned}
$$


where $I_{\epsilon}=\int_{t_{n}}^{t} e^{i \tau / \epsilon} f\left(e^{-i \tau / \epsilon} w_{\epsilon}(\tau), v_{\epsilon}(\tau)\right) d \tau$,

$$
\begin{gathered}
R_{i}=\int_{t_{i}}^{t_{i}+2 \pi \epsilon} e^{-i t / \epsilon}\left(f^{I}\left(e^{i t / \epsilon} w_{\epsilon}(t), v_{\epsilon}(t)\right)-f^{I}\left(e^{i t / \epsilon} w_{\epsilon}\left(t_{i}\right), v_{\epsilon}\left(t_{i}\right)\right)\right) d t \\
\sum_{i=0}^{t_{n}}\left|R_{i}\right| \leq \sum_{i=0}^{t_{n}} 2 \pi \bar{L}\|f\|_{\infty} \epsilon^{2} \leq 2 \pi \bar{L} T^{\prime}\|f\|_{\infty} \epsilon
\end{gathered}
$$

and $\left|I_{\epsilon}\right| \leq \epsilon|| f \|_{\infty}$.

The main summation

$$
\begin{aligned}
\sum_{i=0}^{n} \int_{t_{i}}^{t_{i}+2 \pi \epsilon} & e^{-i t / \epsilon} f\left(e^{-i t / \epsilon} w_{\epsilon}\left(t_{i}\right), v_{\epsilon}\left(t_{i}\right)\right) d t \\
& =\sum_{i=0}^{n} \epsilon \int_{0}^{2 \pi} e^{-i \theta} f\left(e^{i \theta} w_{\epsilon}\left(t_{i}\right), v_{\epsilon}\left(t_{i}\right)\right) d \theta \\
& =\sum_{i=0}^{n} 2 \pi \epsilon \cdot \frac{1}{2 \pi} \int_{0}^{2 \pi} e^{-i \theta} f\left(e^{i \theta} w_{\epsilon}\left(t_{i}\right), v_{\epsilon}\left(t_{i}\right)\right) d \theta
\end{aligned}
$$

is a Riemann sum of $\int_{t_{0}}^{t} \bar{f}\left(w_{\epsilon}, v_{\epsilon}\right) d \tau$. Hence $I_{1} \leq C_{I_{1}} \epsilon$.

Similarly,

$$
\begin{aligned}
\int_{t_{0}}^{t} f^{I I}\left(e^{i \tau / \epsilon} w_{\epsilon}(\tau), v_{\epsilon}(\tau)\right) d \tau \\
\quad=\sum_{i=0}^{n} \int_{t_{i}}^{t_{i}+2 \pi \epsilon} f^{I I}\left(e^{i t / \epsilon} w_{\epsilon}\left(t_{i}\right), v_{\epsilon}\left(t_{i}\right)\right) d t+\tilde{R}_{i}+I I_{\epsilon},
\end{aligned}
$$

with $\sum_{i=0}^{t_{n}}\left|\tilde{R}_{i}\right| \leq \epsilon C_{3}$ and $\left|I I_{\epsilon}\right| \leq \epsilon C_{4}$, and

$$
\sum_{i=0}^{n} \int_{t_{i}}^{t_{i}+2 \pi \epsilon} f^{I I}\left(e^{i t / \epsilon} w_{\epsilon}\left(t_{i}\right), v_{\epsilon}\left(t_{i}\right)\right) d t
$$

is a Riemann sum for $\int_{t_{0}}^{t} \bar{f}^{I I}\left(w_{\epsilon}, v_{\epsilon}\right) d \tau$.

Therefore, for $t_{0} \leq t \leq T^{\prime}, I_{1}+I_{2} \leq C \epsilon$ for some constant $C$.

In the remainder of this section, we assume that the function $f^{I}$ in (3.6) has the property:

$$
f^{I}(x, y, t)=f^{I}\left(e^{i \theta} x, y, t\right), \quad \forall \theta \in \mathbb{R} .
$$

The following lemma shows how well a kernel in $\mathbb{K}^{p, q}([-1,0])$ estimates the effective forces if given the exact data. For convenience, we drop the $\epsilon$ subscript in $x_{\epsilon}$ and $y_{\epsilon}$.

Lemma 3.11. Let $(x, y)$ solve the equations (3.6) for $t_{n} \leq t \leq \eta$ with initial data $x\left(t_{n}\right)=\bar{w}^{n}$ and $y\left(t_{n}\right)=\bar{v}^{n}$, and let $\bar{f}^{I}, \bar{f}^{I I}$ be defined in (3.9). Let $w(t)=$ $e^{i\left(t-t_{n}\right) / \epsilon} x(t)$ and $v(t)=y(t)$. Then $(w, v)$ solve equations (3.7). We have the estimates

$$
\begin{aligned}
E_{k e r}\left(\bar{w}^{n}, \bar{v}^{n}, t_{n} ; f_{\epsilon}^{I}\right): & =\left\|K_{\eta} * f_{\epsilon}^{I}(x, y, \cdot)\left(t_{n}\right)-\bar{f}^{I}\left(\bar{w}^{n}, \bar{v}^{n}\right)\right\| \\
& \leq C_{I} \eta\left(\frac{\epsilon}{\eta}\right)^{q}\left\|K^{(q)}\right\|_{\infty}+C \epsilon^{q-1} / \eta^{q}
\end{aligned}
$$


and

$$
\begin{aligned}
E_{k e r}\left(\bar{w}^{n}, \bar{v}^{n}, t_{n} ; f^{I I}\right): & =\left\|K_{\eta} * f^{I I}(u, v)\left(t_{n}\right)-\bar{f}^{I I}\left(\bar{w}\left(t_{n}\right), \bar{v}\left(t_{n}\right)\right)\right\| \\
& \leq C_{I I}\left(\frac{\epsilon}{\eta}\right)^{q}\|K\|_{W^{1, q}}+\eta C_{f^{I I}}\left(\left\|f^{I}\right\|_{\infty}+\left\|f^{I I}\right\|_{\infty}\right)
\end{aligned}
$$

for some constants $C_{I}$ and $C_{I I}$ independent of $\epsilon$ and $\eta$, and $C_{f^{I I}}$ is a Lipschitz constant of $f^{I I}$.

Proof. We first derive the bound on $E_{k e r}\left(\bar{w}^{n}, \bar{v}^{n}, t_{n} ; f^{I I}\right)$. With $w(s)=w\left(t_{n}\right)+$ $\left(s-t_{n}\right) f^{I}\left(\tilde{w}_{n}, \tilde{t}^{n}\right)$, where $\tilde{w}_{n}$ comes from the mean value theorem, we have

$$
\begin{aligned}
K_{\eta} * g(u, v)\left(t_{n}\right)= & \int_{t_{n}}^{t_{n}+\eta} K_{\eta}(t-s) f^{I I}\left(e^{i s / \epsilon} w\left(t_{n}\right), v\left(t_{n}\right)\right) d s \\
& +\int_{t_{n}}^{t_{n}+\eta} K_{\eta}(t-s)\left(s-t_{n}\right)\left(e^{i s / \epsilon} \partial_{1} f^{I I} f^{I}+\partial_{2} f^{I I} f^{I I}\right) d s,
\end{aligned}
$$

where $\partial_{1} f^{I I}$ and $\partial_{2} f^{I I}$ denote the partial derivatives of $f^{I I}$ with respective to its first and second arguments, respectively. Thus the second integral is bounded above by $\eta C_{f^{I I}}\left(\left\|f^{I}\right\|_{\infty}+\left\|f^{I I}\right\|_{\infty}\right)$, and $C_{f^{I I}}$ is a Lipschitz constant of $f^{I I}$. Let $\tilde{f}^{I I}(t)=f^{I I}\left(e^{i t} \bar{w}^{n}, \bar{v}^{n}\right)$. Then $\tilde{f}^{I I}$ is a $2 \pi$ periodic function. Furthermore, we notice that

$$
\frac{1}{2 \pi} \int_{0}^{2 \pi} \tilde{f}^{I I}(t) d t=\frac{1}{2 \pi} \int_{0}^{2 \pi} f^{I I}\left(e^{i t} \bar{w}^{n}, \bar{v}^{n}\right) d t=\bar{f}^{I I}\left(\bar{w}^{n}, \bar{v}^{n}\right)=: \bar{f}_{n}^{I I}
$$

and that $\tilde{f}^{I I}(t)-\bar{f}_{n}^{I I}$ is a $2 \pi$ periodic function with zero average. Thus by Lemma 2.2, we have

$$
\begin{aligned}
\int_{t_{n}}^{t_{n}+\eta} K_{\eta}(t-s) f^{I I}\left(e^{i s / \epsilon} w\left(t_{n}\right), v\left(t_{n}\right)\right) d s= & \int_{t_{n}}^{t_{n}+\eta} K_{\eta}(t-s)\left(\tilde{f}^{I I}(s)-\bar{f}_{n}^{I I}\right) d s \\
& +\int_{t_{n}}^{t_{n}+\eta} K_{\eta}(t-s) \bar{f}_{n}^{I I} d s \\
= & C_{I}\left(\frac{\epsilon}{\eta}\right)^{q}\|K\|_{W^{1, q}}+\bar{f}_{n}^{I I} .
\end{aligned}
$$

Therefore, we have

$$
\left\|K_{\eta} * f^{I I}(x, y)\left(t_{n}\right)-\bar{f}^{I I}\left(\bar{w}^{n}, \bar{v}^{n}\right)\right\| \leq C_{I}\left(\frac{\epsilon}{\eta}\right)^{q}\|K\|_{W^{1, q}}+\eta L_{g}\left(\left\|f^{I}\right\|_{\infty}+\left\|g^{I I}\right\|_{\infty}\right) .
$$

We now prove the first inequality. Since $x(t)=\exp \left(i\left(t-t_{n}\right) / \epsilon\right) \bar{w}^{n}+\int_{t_{n}}^{t} \exp (i(t-$ $s) / \epsilon) f^{I}(x(s), y(s)) d s$,

$$
\begin{aligned}
K_{\eta} * f_{\epsilon}\left(t_{n}\right)= & \frac{i}{\epsilon} K_{\eta} *\left(e^{i \epsilon^{-1}\left(\cdot-t_{n}\right)} \bar{w}^{n}+\int_{t_{n}} e^{i \epsilon^{-1}(\cdot-s)} f^{I}(x(s), y(s)) d s\right)\left(t_{n}\right) \\
& +K_{\eta} * f^{I}(x, y)\left(t_{n}\right) \\
= & I_{1}+I_{2}+K_{\eta} * f^{I}(x, y)\left(t_{n}\right)
\end{aligned}
$$


where $I_{1}=\frac{i}{\epsilon} \bar{w}^{n} K_{\eta} * \exp \left(i \epsilon^{-1}\left(\cdot-t_{n}\right)\right)$, and $I_{2}$ is the second term in the convolution. Thus $\left\|I_{1}\right\| \leq C_{0} \epsilon^{q-1} / \eta^{q}$ by Lemma 2.2. Integration by parts shows

$$
\begin{aligned}
I_{2}= & \frac{i}{\epsilon} K_{\eta} *\left(\int_{t_{n}} e^{i \epsilon^{-1}(\cdot-s)} f^{I}(x(s), y(s)) d s\right)\left(t_{n}\right) \\
= & -K_{\eta} * f^{I}(x, y)\left(t_{n}\right)+e^{-i t_{n} / \epsilon} f^{I}\left(\bar{w}^{n}, \bar{v}^{n}\right) K_{\eta} * e^{i \cdot / \epsilon} \\
& \quad+K_{\eta} *\left(\int_{t_{n}}^{t} e^{i \epsilon^{-1}(t-s)} \frac{d}{d s} f^{I}(x(s), y(s)) d s\right) .
\end{aligned}
$$

Notice that the first term cancels with the last term in (3.10). By Lemma 2.2, the second term $\mid \exp \left(-i \epsilon^{-1} t_{n} f^{I}\left(\bar{w}^{n}, \bar{v}^{n}\right) K_{\eta} * e^{i \epsilon^{-1} t}\left|\leq C_{K}\right| f^{I}\left(\bar{w}^{n}, \bar{v}^{n}\right) \mid \epsilon^{q} / \eta^{q}\right.$. We now analyze the last term:

$$
\begin{aligned}
I I & =K_{\eta} *\left(\int_{t_{n}}^{t} e^{i(t-s) / \epsilon} \frac{d}{d s} f^{I}(u(s), v(s)) d s\right)(\xi) \\
& =\int_{s=t_{n}}^{t_{n}+\eta} \int_{t=s}^{t_{n}+\eta} K_{\eta}(\xi-t) e^{i \epsilon^{-1} t} e^{-i \epsilon^{-1} s} b(s) d t d s
\end{aligned}
$$

where

$$
\begin{aligned}
b(s) & =\frac{d}{d s} f^{I}(x(s), y(s)) \\
& =\frac{d}{d s} f^{I}(w(s), v(s)) \quad\left(w(s)=e^{i\left(s-t_{n}\right) / \epsilon} x(s), f(x, y)=f(w, v)\right) \\
& =\partial_{1} f^{I}(w, v) e^{-i \epsilon^{-1} t} f^{I}(w, v)+\partial_{2} f^{I}(v, s) f^{I I}(x, y) .
\end{aligned}
$$

Switching the order of integrations, we have

$$
|I I| \leq \int_{s=t_{n}}^{t_{n}+\eta}|b(s)| d s\left|\int_{t=t_{n}}^{t_{n}+\eta} K_{\eta}\left(t_{n}-t\right) e^{i \epsilon^{-1} t} d t\right| \leq \operatorname{const} \eta\|b\|_{\infty}\left(\frac{\epsilon}{\eta}\right)^{q}\left\|K^{(q)}\right\|_{\infty} .
$$

Hence,

$$
\left\|K_{\eta} * f_{\epsilon}^{I}(u, v, \cdot)\left(t_{n}\right)\right\| \leq C_{I} \eta\left(\frac{\epsilon}{\eta}\right)^{q}\left\|K^{(q)}\right\|_{\infty}
$$

Now we analyze the local errors made in one step of the HMM-FE-* schemes by grouping the errors according to how they are committed in the HMM process. We first remind the readers of the notation.

Let $U\left(t_{n}\right)=\left(X\left(t_{n}\right), Y\left(t_{n}\right)\right)$ be the solution of the effective equation (1.4) at grid nodes $t_{n}=t_{0}+n H$, with initial condition $U\left(t_{0}\right)=U_{0}=\left(X_{0}, Y_{0}\right)$. Let $U^{n}=$ $\left(X^{n}, y^{n}\right)$ denote the numerical approximations for $U\left(t_{n}\right)$ produced by the chosen HMM-FE- $\Upsilon$ scheme; here $\Upsilon$ is the selected micro-solver. Define $E^{n}=U\left(t_{n}\right)-U^{n}$. Assuming that $\bar{f}$ can be evaluated with no error, we first have the standard relation:

$$
E^{n+1}=E^{n}+H\left(\bar{f}\left(U\left(t_{n}\right)\right)-\bar{f}\left(U^{n}\right)\right)+\mathcal{E}_{\text {macro }}^{n}=\left(I+H D \bar{f}_{n}\right) E^{n}+\mathcal{E}_{\text {macro }}^{n},
$$

where $\mathcal{E}_{\text {macro }}^{n}$ is the usual local truncation error of the Forward Euler scheme, and $D \bar{f}_{n}$ is the Jacobian matrix obtained through the mean value theorem. Next, in HMM schemes, since $\bar{f}$ is actually approximated through Step 1 in the algorithm described in Section 2.1, there are further sources of errors coming from microscopic processes from Step 1(a)-(d). Denote by $\mathcal{E}_{H M M}$ the errors made altogether from Step 1(a)-1(d). It becomes clear that $\mathcal{E}_{H M M}=\mathcal{E}_{H M M}^{(a)}+\mathcal{E}_{H M M}^{(\Upsilon)}$, where $\mathcal{E}_{H M M}^{(a)}$ 
corresponds to the analytical errors committed and $\mathcal{E}_{H M M}^{(\Upsilon)}$ corresponds to the discretization error. We have been analyzing $\mathcal{E}_{H M M}^{(a)}$ in this paper, and the bounds appear in the theorems in Section 2 and Lemma 3.11 $\mathcal{E}_{H M M}^{(\Upsilon)}=\mathcal{E}_{\text {micro }}^{(\Upsilon)}+\mathcal{E}_{\text {quad }}$ is the sum of the global error in evaluating $f_{\epsilon}$ accumulated in Step 1(b), and the quadrature error $\mathcal{E}_{\text {quad }}$ corresponds to Step $1(\mathrm{~d})$. The justification of using $R=Q=I$, the identity operator, is absorbed into $\mathcal{E}_{H M M}^{(a)}$. Here, it is understood that the terms in $\mathcal{E}_{H M M}=\mathcal{E}_{H M M}^{n}$ actually depend on $U\left(t_{n}\right), f_{\epsilon}$ and $t_{n}$. We remark that there are problems not considered in this paper in which $R$ or $Q$ require further analytical and numerical approximations.

In summary, for an HMM-FE- $\Upsilon$ scheme, we have

$$
E^{n+1}=\left(I+H D \bar{f}_{n}\right) E^{n}+H \mathcal{E}_{H M M}^{n},
$$

where $\mathcal{E}_{H M M}^{n}=\mathcal{E}_{H M M}^{(a)}+\mathcal{E}_{\text {micro }}^{(\Upsilon)}+\mathcal{E}_{\text {quad }}$. Writing $A_{n}=\left(I+H D \bar{f}_{n}\right)$ for convenience, we have

$$
E^{n+1}=\Pi_{j=0}^{n} A_{j} E^{0}+\sum_{j=1}^{n+1} \Pi_{k=j}^{n} A_{k}\left(H \mathcal{E}_{H M M}^{j-1}+\mathcal{E}_{\text {macro }}^{j-1}\right)
$$

In the Forward Euler scheme, $\left\|\mathcal{E}_{\text {macro }}\right\| \leq C_{\text {trunc }} H^{2}$. Assuming that the macrosolver is stable, i.e. $\left\|A_{j}\right\|$ is bounded,

$$
\left\|A_{j}\right\| \leq C_{a m p 1} e^{\mu H}, \text { for } 0 \leq j \leq n .
$$

Then if we have $\left\|\mathcal{E}_{H M M}^{j-1}\right\| \leq C_{H m m} H$ for some $\epsilon \leq \epsilon_{0}(H)$, and $\eta \leq \eta_{0}(H)$, and $\epsilon_{0}, \eta_{0}$ decrease with $H$, we have a bound on the global error of the macro-solver in $H$.

Assuming that we use a 4th-order Runge-Kutta scheme in Step 1(b), in the time interval $\left[t_{n}, t_{n}+\eta\right]$, the global error in the approximation of $u_{\epsilon}$ is $C_{r k 4} h^{4} \eta \epsilon^{-5}$, where the last factor $\epsilon^{-5}$ comes from $d^{(5)} u_{\epsilon} / d t^{(5)}$; therefore the error in evaluating $f_{\epsilon}$, i.e. $\mathcal{E}_{\text {micro }}^{(\Upsilon)}$, becomes $\tilde{C}_{r k 4} h^{4} \eta \epsilon^{-6}$.

By Lemma 3.11 .

$$
\left\|\mathcal{E}_{H M M}^{(a)}\right\| \leq \tilde{C} \epsilon^{q-1} / \eta^{q}+\eta C_{f^{I I}}\left(\left\|f^{I}\right\|_{\infty}+\left\|f^{I I}\right\|_{\infty}\right) .
$$

If we take the composite trapezoidal rule as our numerical quadrature, for general smooth functions, $\left\|\mathcal{E}_{\text {quad }}\right\| \leq \tilde{C}_{\text {quad }} h^{2}$. However, due to the regularity of the kernel used, $K \in C_{c}^{q}(\mathbb{R})$, our integrand is smooth and periodic, and therefore its Fourier spectrum decays very fast. Since the trapezoidal rule is exact for $e^{2 \pi i \cdot l x}$ for $l=$ $0, \ldots, 2 m,\left\|\mathcal{E}_{\text {quad }}\right\|$ is typically very small and negligible.

Assume that for $T_{0} \leq t \leq T_{1}$, the analytical solution $U=(X, Y)$ of the effective equations (3.9) is bounded by $\bar{M}:|X(t)|+|Y(t)| \leq \bar{M}$, and that $\mid X^{k}\|+\| Y^{k} \| \leq$ $\bar{M}+m^{\prime}$ for $k=0,1, \ldots, n<N$ and $T_{0}+N h \leq T_{1}$. Assume in addition that we start with the exact initial conditions. The error (3.11) is bounded by

$$
\begin{aligned}
\left|E^{n+1}\right| & =\sum_{j=1}^{n+1} \Pi_{k=j}^{n}\left\|A_{k}\right\|\left(H\left\|\mathcal{E}_{H M M}^{j-1}\right\|+\left\|\mathcal{E}_{\text {macro }}^{j-1}\right\|\right) \\
& \leq C_{1} e^{\mu\left(T_{1}-T_{0}\right)} H\left(1+\sum_{j=1}^{n+1}\left\|\mathcal{E}_{H M M}^{j-1}\right\|\right)
\end{aligned}
$$


For $\eta=C_{\eta} H^{-1 / q} \epsilon^{(q-1) / q}, h=C_{h} \eta^{-1 / 4} H^{1 / 4} \epsilon^{1+2 / 4},\left\|\mathcal{E}_{H M M}^{j}\right\| \leq C_{\bar{M}+m^{\prime}} H$, for $0 \leq$ $j \leq n$, and $\left|E^{n+1}\right| \leq C \exp \left(T_{1}-T_{0}\right) H$.

With $H$ and $T_{1}-T_{0}$ sufficiently small, we have

$$
\left\|X^{n+1}\right\|+\left\|Y^{n+1}\right\| \leq \bar{M}+\left\|E^{n+1}\right\| \leq \bar{M}+m^{\prime} .
$$

Thus we can iterate our arguments and obtain the following theorem:

Theorem 3.12. Given an HMM-FE-rk4 scheme, let $U^{n}=\left(X^{n}, Y^{n}\right)$ be the solution at $t_{n}=T_{0}+n H$, computed by this scheme applied to (3.6), and let $U(t)$ be the analytical solution to (3.9). Let $H, h, \eta$ be respectively the macro step size, micro step size, and the length of each microscale evaluation. Then there are constants $C$ such that for $h \leq h_{0}(H, \epsilon), \eta \leq \eta_{0}(H)<H, H<H_{0}$, and for $t \in\left[T_{0}, T_{1}\right]$, the global error $E^{n}:=U\left(t_{n}\right)-U^{n}$ is bounded above by $H$ :

$$
\left|X^{n}-X_{0}\right| \leq C H
$$

and

$$
\left|Y^{n}-Y\left(t_{n}\right)\right| \leq C H,
$$

for $0 \leq n \leq N$, where $T_{0}+N H=T_{1}$.

3.3. Complexity estimates. In the previous subsection, we see in an HMM-FErk4 scheme for the oscillatory problem (3.9), how the kernel support size $\eta$ and the step size $h$ for the micro-solver should scale (up to constants) with the macro-solver step size $H$. This is done through balancing different errors to the same order in $H$. We now describe this balancing of errors for oscillatory problems of a general HMM- $\mathcal{X}-\Upsilon$ scheme. The dissipative case is analyzed similarly.

Assume that the macro-solver $\mathcal{X}$ is an $s$-th order accurate scheme, the microsolver is $r$-th order accurate, and $K_{\eta} \in \mathbb{K}^{p, q}$ is used. We want our kernel estimation error $\mathcal{E}_{H M M}=\| \bar{f}\left(U^{n}\right)-\sum_{j} \omega_{j} K_{j} f\left(u_{\epsilon}\left(t_{n}\right) \|\right.$ to be bounded by $C_{K} H^{s}$ so that it is comparable to the local truncation error of the given macro-scheme. Similar to what we have seen previously, the error in approximating $u_{\epsilon}$ in Step 1(b) is

$$
C h^{r} \eta \epsilon^{-(r+1)}
$$

where the factor $\epsilon^{-(r+1)}$ comes from $d^{(r+1)} u_{\epsilon} / d t^{(r+1)}$, and thus

$$
\left|\mathcal{E}_{\text {micro }}^{(\Upsilon)}\right| \leq C\left\|\frac{\partial f_{\epsilon}}{\partial u}\right\|_{\infty} \eta h^{r} \epsilon^{-(r+1)} \leq \tilde{C} \eta h^{r} \epsilon^{-(r+2)} .
$$

Hence we need $\eta h^{r} \epsilon^{-(r+2)}=C^{\prime} H^{s}$, so omitting the constant,

$$
h=\eta^{-1 / r} H^{s / r} \epsilon^{1+2 / r} .
$$

From Section 2, we know that, for suitable problems, the force estimation error of a kernel in $\mathbb{K}^{p, q}$ is

$$
\mathcal{E}_{H M M}^{(a)} \leq C_{1} \eta^{p}+C_{g_{\epsilon}}\left(\frac{\epsilon}{\eta}\right)^{q} .
$$

$C_{g_{\epsilon}}$ in our case is proportional to $\left\|\partial f_{\epsilon} / \partial u_{\epsilon}\right\|_{\infty}$; thus,

$$
C_{g_{\epsilon}}\left(\frac{\epsilon}{\eta}\right)^{q}=C \frac{\epsilon^{q-1}}{\eta^{q}}
$$

Omitting the constants, the two terms in (3.12) are of the same order when $\eta$ is proportional to $\eta^{*}(\epsilon)=\epsilon^{(q-1) /(p+q)}$, and the $\epsilon^{q-1} / \eta^{q}$ term dominates $\eta^{p}$ for 
$\eta<\eta^{*}(\epsilon)$. To minimize the number of microscale time steps, we want $\eta$ to be small. Hence, we want to scale $\eta<\eta^{*}(\epsilon)$ such that the dominating term

$$
\frac{\epsilon^{q-1}}{\eta^{q}} \sim H^{s} \Longrightarrow \eta=H^{-s / q} \epsilon^{(q-1) / q}
$$

The constraint $\eta<\eta^{*}$ is satisfied if

$$
H>\epsilon^{-\omega}
$$

where $\omega>0$ is a constant found by balancing all the exponents involving positive integers $p$ and $q$.

Hence, with $\eta=H^{-s / q} \epsilon^{1-1 / q}$,

$$
\text { \# micro timesteps }=\frac{\eta}{h}=H^{-\alpha} \epsilon^{-\beta},
$$

where

$$
\alpha=\frac{r s+q s+s}{q r}, \quad \beta=\frac{q+r+1}{q r}>0,
$$

since $p, q, r$ are positive integers, $0<\beta<1$. Assuming that $1 / H$ macro steps are needed to reach $T$, the total complexity is

$$
\text { \# total HMM steps }=H^{-1-\alpha} \epsilon^{-\beta} \leq C \text {. }
$$

Example 3.13. In the following, we give a really conservative error estimate for an HMM-FE-rk4 method using a kernel in $\mathbb{K}^{2,5}(s=1, r=4, p=2, q=5)$.

- Global error in $\eta$-interval: rk4 gives $h^{4} \eta \epsilon^{-5}$. The last factor $\epsilon^{-5}$ is from $d^{5} z / d t^{5}$.

- $E_{4}$ scaled with $H$ gives $\eta h^{4} \epsilon^{-6}=H$.

- Error in the approximation of $\bar{f}: \epsilon^{4} / \eta^{5}=H$.

Thus, $\eta=\epsilon^{4 / 5} H^{-1 / 5}$ and correspondingly $h=\epsilon^{6 / 5} H^{6 / 5}$. An estimate for the number of flops for the HMM-FE-rk4 method with $\eta / h$ micro steps for each of the $N \sim 1 / H$ macro steps is

$$
\text { \# of Flops }=N \frac{\eta}{h}=\epsilon^{-1 / 2} H^{-3 / 2} .
$$

We compare this particular HMM-FE-rk4 method to other methods. Any explicit methods will need a step size of the order of $\epsilon$ to resolve the oscillations and to be stable. The complexity then is proportional to $\epsilon$, which makes the computation impossible, let alone other properties such as, possibly, unnecessary damping in the solution. This evidence suggests that our HMM-FE-rk4 is superior.

\section{Analytical and numerical EXamples}

In this section, we apply our schemes to a few examples. For each fixed $\epsilon$, our numerical tests verify the results for the HMM schemes developed in the previous section.

We set up our numerical simulations as follows. The numerical approximations are computed in the time interval $\left[0, T_{1}\right]$ using a macro step size $H \in\left\{T_{1} / n_{j}: n_{j}=\right.$ $10+10 j, j=0,1,2, \ldots, 7\}$. We use $H_{j}$ to denote $T_{1} / n_{j}, h_{j}$ for the corresponding micro step size, and $\eta_{j}$ for the kernel support size. With a given $\epsilon$, and the quadruple $(p, q, r, s)$ representing that a kernel in $\mathbb{K}^{p, q}(I)$ is used in Step $1(\mathrm{~d})$, and an $s$-th 
order macro-solver and $r$-th order micro-solver are used in the chosen HMM scheme, we determine $\eta_{j}$ and $h_{j}$ according to our discussion earlier:

$$
\begin{gathered}
\eta_{j}=C_{\eta} H^{-s / q} \epsilon^{1-1 / q}, \\
h_{j}=C_{h} \eta^{-1 / r} H^{s / r} \epsilon^{1+2 / r} .
\end{gathered}
$$

We compute the absolute errors in the $Y$ component defined by

$$
e_{L^{1}}^{(j)}=\sum_{n=1}^{T_{1} / H_{j}}\left|Y^{n}-Y\left(n H_{j}\right)\right| H_{j}
$$

and

$$
e_{\infty}^{(j)}=\max _{1 \leq n \leq T_{1} / H_{j}}\left|Y^{n}-Y\left(n H_{j}\right)\right| .
$$

Finally, these errors are plotted using loglog plots in each of the following examples. In the following subsections, AB2 stands for the 2nd order Adam-Bashforth scheme, LF for Leapfrog, FE for Forward Euler scheme, and rk4 stands for the common 4thorder explicit Runge-Kutta scheme.

\subsection{A stiff oscillatory example.}

$$
\left\{\begin{array}{l}
\dot{x}=i \epsilon^{-1}(x-y)+i(y-t)+1 \\
\dot{y}=i(y-t)+|x-y|^{2}
\end{array},\left(\begin{array}{l}
x_{0} \\
y_{0}
\end{array}\right)=\left(\begin{array}{l}
2 \\
1
\end{array}\right),\right.
$$

with solution

$$
\left\{\begin{array}{l}
x=e^{i \epsilon^{-1} t}+e^{i t}+t, \\
y=e^{i t}+t
\end{array}\right.
$$

Before applying HMM ODE schemes to this system, we remark that this system is in the class of nonlinear equations that we have considered in the previous section. System (4.1) after a linear transformation $(w, y)=(x-y, y)$, becomes

$$
\left\{\begin{array}{l}
\dot{w}=i \epsilon^{-1} w-|w|^{2}+1 \\
\dot{y}=|w|^{2}
\end{array},\left(\begin{array}{l}
w_{0} \\
y_{0}
\end{array}\right)=\left(\begin{array}{l}
1 \\
0
\end{array}\right),\right.
$$

which is obviously in the form to which our convergence theory applies. We have mentioned that the averaging approach is invariant of linear transformations, so we can work directly with (4.1). We see in this example that we cannot simply ignore the fast scale variable $x$, since it contributes the dynamics of $y$.

In Figure 4.1, we plot $e_{L^{1}}^{(j)}$ and $e_{\infty}^{(j)}$ for each computation using the macroscopic step size $H_{j}=4 /(10+10 j)$ along with the following settings:

(a) HMM-FE-lf: $K^{\exp }([-1,1]), \delta t_{*}=0,(p, q, r, s)=(1,10,2,1), \epsilon=$ $10^{-3} /(2 \pi), C_{\eta}=5, C_{h}=0.4$

(b) HMM-FE-rk4: $K^{\exp }([-1,1]), \delta t_{*}=0,(p, q, r, s)=(1,10,4,1), \epsilon=$ $10^{-5} /(2 \pi), C_{\eta}=2.6, C_{h}=0.5$

(c) HMM-LF-rk4: $K^{\exp }([-1,1]), \delta t_{*}=0,(p, q, r, s)=(1,10,4,2), \epsilon=$ $10^{-6} /(2 \pi), C_{\eta}=6, C_{h}=2$;

(d) HMM-AB2-rk4: $K^{\exp }([-1,1]), \delta t_{*}=0,(p, q, r, s)=(1,10,4,2), \epsilon=$ $10^{-6} /(2 \pi), C_{\eta}=6, C_{h}=2$.

We remark that since the system is not stiffly dissipative, in Step 1(b), we solve $u_{\epsilon, n}$ in $\left[t_{n}, t_{n}+\delta t_{*}\right]$ by the given equation, and in $\left[t_{n}, t_{n}-\delta t_{*}\right]$ backward in time with the initial condition $u_{\epsilon, n}\left(\tau=t_{n}\right)=U^{n}$. Then the effective force can be evaluated at $t_{n}$ using a symmetric kernel. 


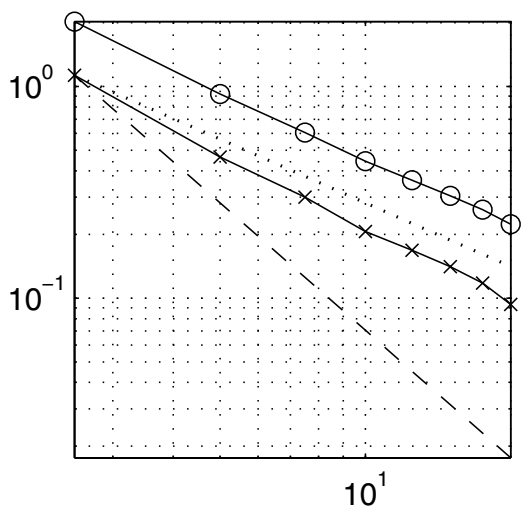

(a) FE-lf

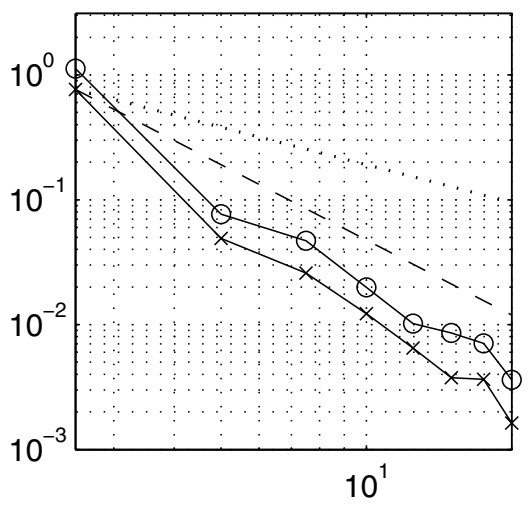

(c) LF-rk4

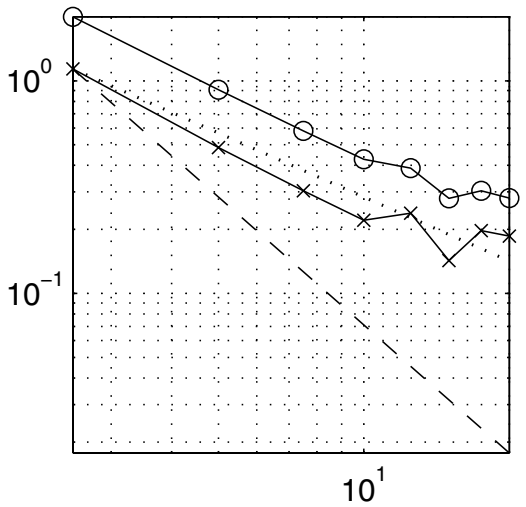

(b) FE-rk4

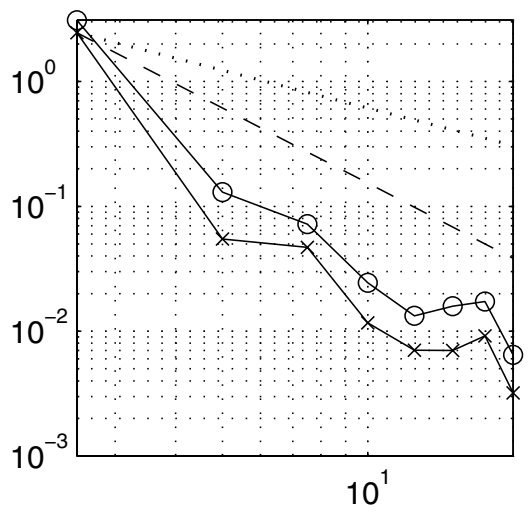

(d) AB2-rk4

Figure 4.1. The horizontal axes $1 / H$. The lines with x-markers are $e_{\infty}^{(j)}$ and the lines with o-markers are $e_{L^{1}}^{(j)}$. The dotted lines are proportional to $H$, and the dashed lines are proportional to $H^{2}$. The detailed settings are provided in the example of Section 4.1.

\subsection{Dissipative systems.}

4.2.1. Stiff system with fast transient. We test the HMM-AB2-rk4 solver (with a 4th-order Runge-Kutta for microscale and the 2nd-order Adam-Bashforth for macroscale) on the following equation:

$$
y^{\prime}=-\epsilon^{-1}(y+\cos t), \quad y(0)=2.0 .
$$

Since the macro-solver is a linear multistep method that requires a uniform step size, we use the nonsymmetric kernel, $K \in \mathbb{K}^{2,3}([-1,0]) \cap C_{c}^{3}([-1,0.2])$, depicted in Figure 1.1. to estimate the effective force at the left end of each microscale evolution.

HMM-AB2a-rk4: $\delta t_{*}=0.6 \eta,(p, q, r, s)=(2,3,4,2), \epsilon=10^{-4}, C_{\eta}=25, C_{h}=$ $0.25, H \in\left\{T_{1} / n_{j}: n_{j}=40+20 j, j=0,1,2, \ldots, 10\right\}, T_{1}=4$. The errors are plotted in Figure 4.2 . 


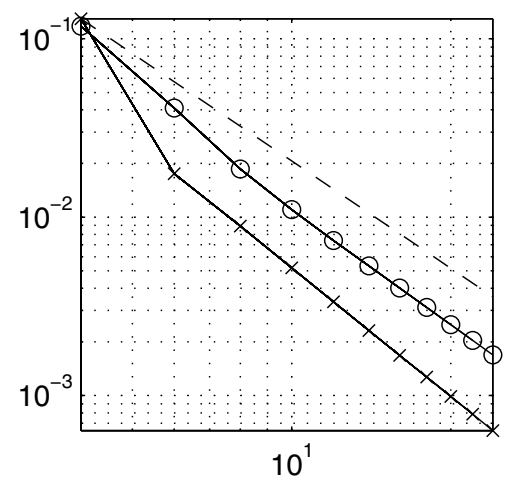

Figure 4.2. HMM-AB2a-rk4. The horizontal axis is $1 / H$. The lines with x-markers are $e_{\infty}^{(j)}$ and the lines with o-markers are $e_{L^{1}}^{(j)}$. The dashed lines are proportional to $H^{2}$. The detailed settings are provided in Section 4.2.1.

\subsubsection{Oscillatory system with transient.}

$$
\left\{\begin{array}{l}
\dot{x}=\epsilon^{-1}\left(i+\frac{5}{2}\left(1-|x-y|^{2}\right)\right)(x-y)+i(y-t)+1 \\
\dot{y}=i(y-t)+|x-y|^{2}
\end{array},\left(\begin{array}{l}
x_{0} \\
y_{0}
\end{array}\right)=\left(\begin{array}{l}
3 \\
1
\end{array}\right) .\right.
$$

We see from the equation that a stiff transient in $x$ would take place whenever $|x-y|$ is not 1 . However, passing the transients, the averaged solution should be identical to that of system (4.1). In the following computations, the macro time step is taken to be $H \in\left\{2 / n_{j}: n_{j}=10+10 j, j=0,1,2, \ldots, 8\right\}$. The corresponding errors are plotted in Figure 4.3.

HMM-AB2-rk4: $K \in \mathbb{K}^{2,3}([-1,0]) \cap C_{c}^{3}([-1,-0.1]), \delta t_{*}=0,(p, q, r, s)=$ $(2,3,4,2), \epsilon=10^{-6} /(2 \pi), C_{\eta}=0.23, C_{h}=10, T_{1}=2$.

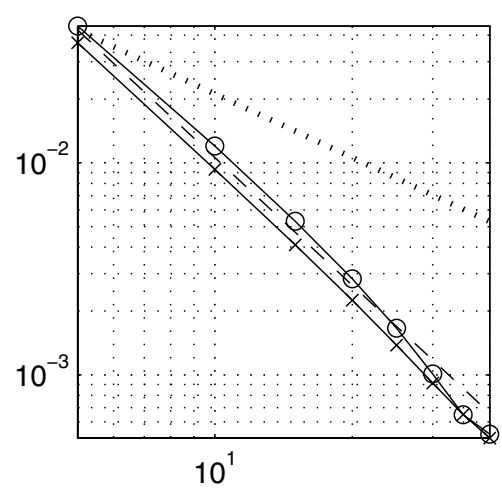

(a) AB2a-rk4

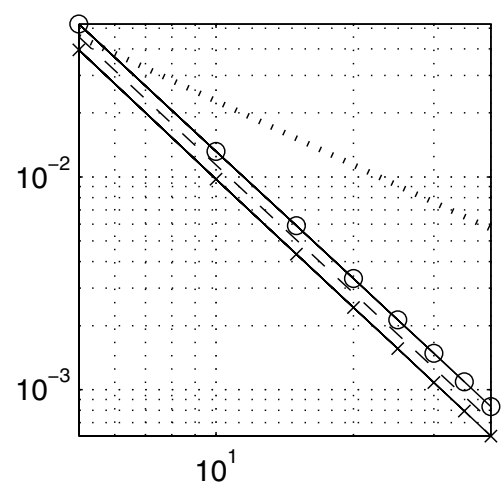

(b) AB2s-rk4

Figure 4.3. Numerical accuracy study of (4.3). The lines with $\mathrm{x}$ markers are $e_{\infty}^{(j)}$ and the lines with o-markers are $e_{L^{1}}^{(j)}$. The dotted lines are proportional to $H$, and the dashed lines are proportional to $H^{2}$. The detailed settings are provided in Section 4.2.2. 
HMM-AB2-rk4: $K(t)=K^{\exp }(t+0.2), \delta t_{*}=0.6 \eta,(p, q, r, s)=(10,1,4,2)$, $\epsilon=10^{-6} /(2 \pi), C_{\eta}=20.3, C_{h}=2, T_{1}=2$. Note that $U$ is constructed at $T_{n}=$ $0.6 \eta+n H$.

4.3. A three-body problem. Let $x=\left(x_{1}, x_{2}, x_{3}\right) \in R^{2} \times R^{2} \times R^{2}$ and let $\left(m_{1}\right.$, $\left.m_{2}, m_{3}\right) \in R^{3}$ denote, respectively, the positions and the masses of the three planets of the system. See Figure 4.4 for an illustration of the setup. The potential energy of this system takes the form:

$$
V(x)=-\sum_{i<j} \frac{m_{i} m_{j}}{\left|x_{i}-x_{j}\right|}=-\left(\frac{m_{1} m_{2}}{\left|x_{1}-x_{2}\right|}+\frac{m_{1} m_{3}}{\left|x_{1}-x_{3}\right|}+\frac{m_{2} m_{3}}{\left|x_{2}-x_{3}\right|}\right) .
$$

The corresponding equations of motion are

$$
m_{i} \ddot{x}_{i}=\nabla_{x_{i}} V(x), \quad i=1,2,3 .
$$

We solve these second-order equations as a system of first-order equations by introducing new dependent variables $v_{i}=\dot{x}_{i}$ and $\dot{v}_{i}=\ddot{x}_{i}$. In our setting, we assume that $m_{2}$ is $O(1)$ and $\mathcal{O}(\epsilon)=m_{1} \ll m_{2} \ll m_{3}=\mathcal{O}(1 / \rho) . x_{3}$ is stationary. We assume that

$$
\left|x_{1}-x_{2}\right|^{2} \sim \epsilon,\left|x_{2}-x_{3}\right|^{2} \sim \rho^{-1} \text { and }\left|x_{1}-x_{3}\right|^{2} \sim \rho^{-1}
$$

so that the equations for $v_{i}$ have only two scales:

$$
\begin{gathered}
\dot{v}_{1}=-\underbrace{m_{2} \frac{\left(x_{1}-x_{2}\right)}{\left|x_{1}-x_{2}\right|^{3}}}_{O(1 / \epsilon)}-\underbrace{m_{3} \frac{\left(x_{1}-x_{3}\right)}{\left|x_{1}-x_{3}\right|^{3}}}_{O(1)}=f^{I}(x)+\frac{1}{\epsilon} g(x), \\
\dot{v}_{2}=-\underbrace{m_{1} \frac{\left(x_{2}-x_{1}\right)}{\left|x_{2}-x_{1}\right|^{3}}}_{O(1)}-\underbrace{m_{3} \frac{\left(x_{2}-x_{3}\right)}{\left|x_{2}-x_{3}\right|^{3}}}_{O(1)}=f^{I I}(x) .
\end{gathered}
$$

Thus, with suitable initial conditions, $m_{1}$ spins around $m_{2}$ in $\epsilon$ time scale while $m_{2}$ orbits around $m_{3}$ in the slow time scale. Essentially, we have two time scales in this problem and the HMM method resolves the trajectory of $m_{2}$ and $m_{1}$ in a short time $\eta$, then takes a big time step. See Figure 4.5 for a snapshot of an actual computation.

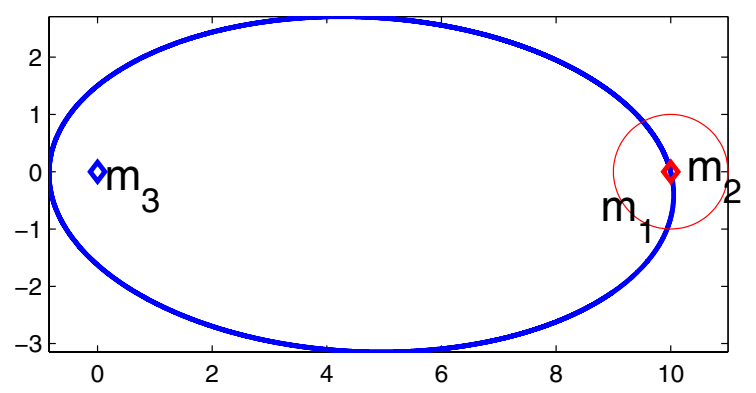

FiguRE 4.4. This is a diagram showing the planar three-body problem. In our setup, $m_{1}$ spins around $m_{2}$ is the $\epsilon$ time scale. 


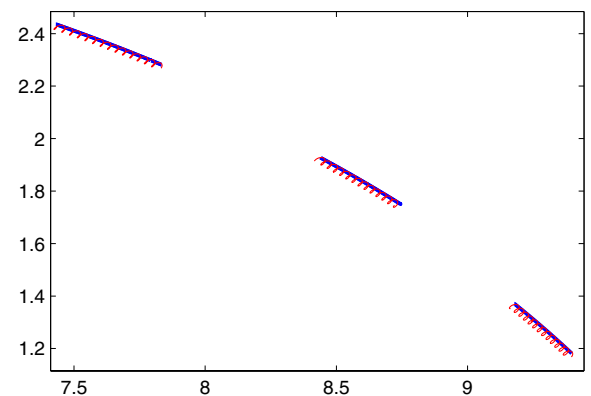

(a)

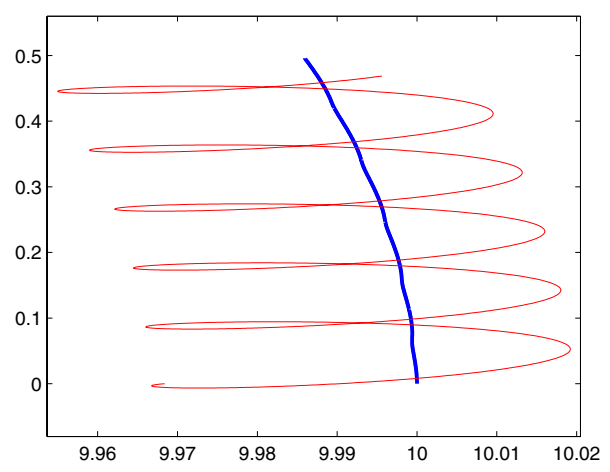

(b)

FigURE 4.5. Snapshots of the computed trajectories

Simulation 1. $\epsilon=10^{-5} /(2 \pi), \rho=10^{-4},\left(m_{1}, m_{2}, m_{3}\right)=(500 * \epsilon, 0.4,5 / \rho), x_{1}(0)=$ $x_{2}(0)+0.5(\sqrt{\epsilon}, \sqrt{\epsilon}), x_{2}(0)=(\sqrt{\rho}, 0), x_{3}(0)=(0,0)$, and $v_{1}(0)=(5,-5), v_{2}(0)=$ $(0,10), v_{1}(0)=(0,0), T_{1}=5.0$.

HMM-LF-rk4: $K^{\exp }, \delta t_{*}=0, C_{\eta}=230 / 2 C_{h}=120, T_{1}=5.0$. We first apply the scheme with different $H$ 's to establish a convergence evidence for this method. Denote by $X_{2}^{L F(H)}$ the position of mass $m_{2}$ at $T_{1}$ constructed this way using macro step size $H$. We obtain $\left|X_{2}^{L F(0.5)}-X_{2}^{L F(0.25)}\right| \doteq 18.29904, \mid X_{2}^{L F(0.25)}-$ $X_{2}^{L F(0.125)}|\doteq 5.47153,| X_{2}^{L F(0.125)}-X_{2}^{L F(0.0625)} \mid \doteq 1.04626$, and from these data, we saw that the difference is deceasing at a rate proportional to $H^{2}$. In Figure 4.6 are plotted the trajectories of $X_{2}^{L F(H)}$ and also the total energy of the system versus $1 / H$, which shows a convergence in total energy at a rate proportional to $H^{2}$.

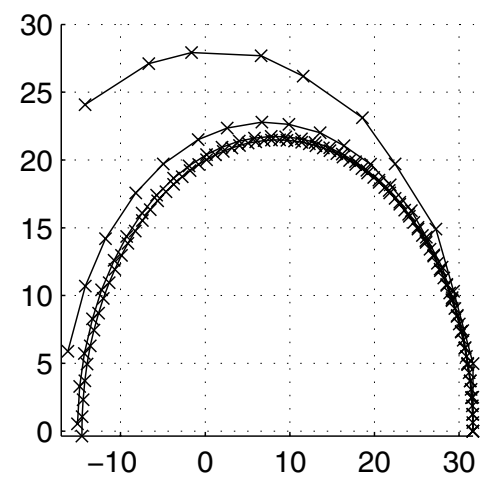

(a) Trajectories

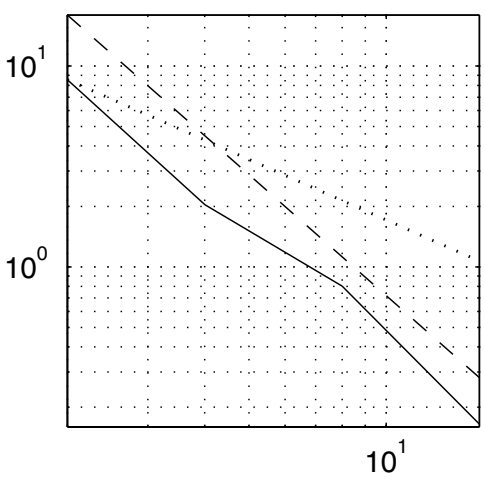

(b) Energy convergence

FiguRE 4.6. Numerical convergence study of the energy simulation 1 of HMM-LF-rk4. The plot on the left shows the difference of the total energy of approximation at $T_{1}$ to the initial energy versus $1 / H . C_{\eta}=230, C_{h}=120, T=5.0$. 

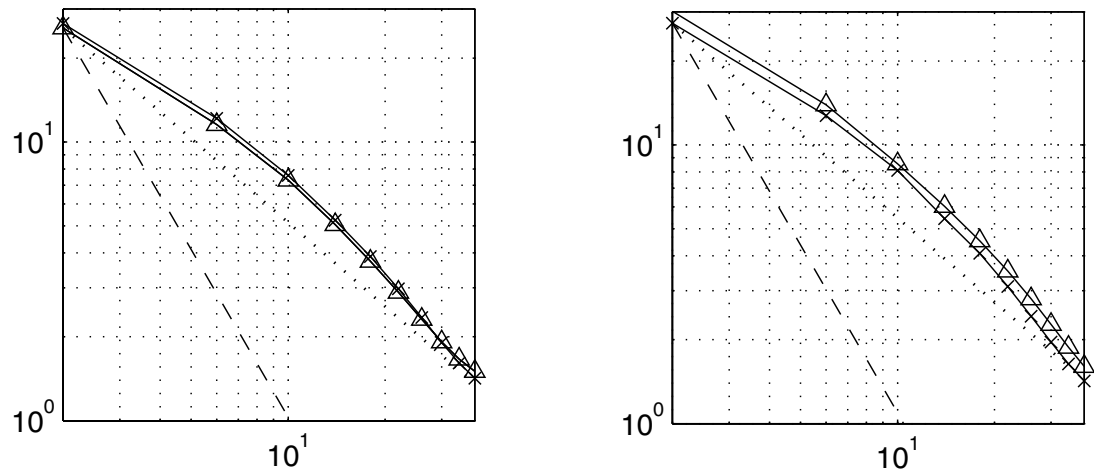

Figure 4.7. Planar three-body problem numerical convergence study of simulation 1 . The curves marked with $\triangle$ are data obtained from HMM-FE-rk 4 with symmetric kernel $K^{\exp }$ (i.e., $\delta t^{*}=0$ ) with backward flow to enforce uniform step sizes. The curves marked with $\times$ are data obtained from HMM-FE-rk4 with symmetric kernel $K^{\exp }$ and variable step sizes. The plot on the left is obtained with $C_{\eta}=200$, and the one on the right with $C_{\eta}=230 / 2$.

In the following set of simulations, we compare the numerical approximations obtained from two different first-order schemes to the $X_{2}^{L F(0.05)}\left(T_{1}\right)$. In our simulations, we ran the following two first-order schemes:

- HMM-FE-rk4: $K^{\exp }([-1,1]): \delta t_{*}=0,(p, q, r, s)=(1,10,4,1), C_{\eta}=230 / 2$ or $200, C_{h}=120, T_{1}=5.0$.

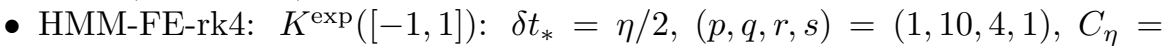
$230 / 2$ or $200, C_{h}=120, T_{1}=5.0$.

The two schemes differ in that one keeps uniform macroscopic time steps and the other one can be considered as a variable time stepping method. We plot $\left|x_{2}-X_{2}^{L F(0.05)}\right|$ versus $1 / H$ in Figure 4.7.

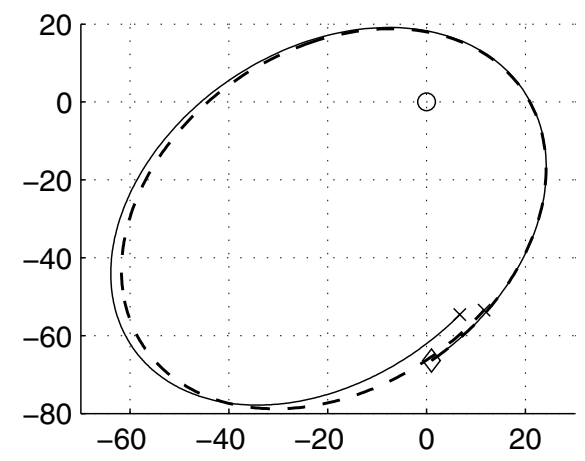

FiguRe 4.8. Planar three-body problem numerical convergence study of simulation 2 . The circle is placed at the position of $x_{3}$. The diamond and cross represent, respectively, the $x_{2}(0)$ and $x_{2}(80)$. The dashed line shows the orbit if $m_{1}=0$. 
Simulation 2. $\epsilon=10^{-5} /(2 \pi), \rho=10^{-4},\left(m_{1}, m_{2}, m_{3}\right)=(500 * \epsilon, 5.87,1 / \rho), x_{1}(0)=$ $x_{2}(0)+0.5(\sqrt{\epsilon}, \sqrt{\epsilon}), x_{2}(0)=(1,-2.1 \sqrt{\rho}), x_{3}(0)=(0,0)$, and $v_{1}(0)=v_{2}(0)+(60,0)$, $v_{2}(0)=(2.5,2.2), v_{3}(0)=(0,0), H=0.2, C_{\eta}=200 / 2, C_{h}=100$.

HMM-LF-rk4: $K^{\exp }$ and $\delta t_{*}=0, T_{1}=80$. See Figure 4.8 .

\section{SUmmary}

In this paper, we introduce and analyze a new class of numerical algorithms for stiff ODE systems based on the HMM framework. We study the stability and convergence of the HMM schemes and present a few numerical computations on stiff systems using either Runge-Kutta or linear multistep HMM schemes. The analysis also covers some existing classes of methods that use variable step sizes. An important component in the new effective force estimation is accomplished through the convolution of the data with certain classes of compactly supported kernels. In particular, we show that a general class of highly stiff systems with oscillatory solutions, for the first time can be practically approximated with an explicit technique that requires fewer functional evaluations than the number of oscillations.

We point out that the HMM methods are not limited to the few simple schemes that we listed above. We show that one can use this methodology to build an HMM scheme for a hierarchy of scales. One can customize and fine tune the numerical approximation scheme in each scale more or less independently from the other scales. For example, one can naturally adopt symplectic schemes for Hamiltonian systems. The HMM framework provides a systematic way to build a scheme that is suitable for a given system.

\section{ACKNowledgments}

The authors thank Weinan E, Yannis Kevrekidis, and Heinz-Otto Kreiss for many useful conversations and the reviewers for their constructive criticisms.

\section{REFERENCES}

[1] V. I. Arnol'd. Mathematical methods of classical mechanics. Springer-Verlag, New York, 1989. Translated from the 1974 Russian original by K. Vogtmann and A. Weinstein, Corrected reprint of the second (1989) edition. MR1345386 (96c:70001)

[2] N. N. Bogoliubov and Y. A. Mitropolsky. Asymptotic methods in the theory of non-linear oscillations. Translated from the second revised Russian edition. International Monographs on Advanced Mathematics and Physics. Hindustan Publishing Corp., Delhi, Gordon and Breach Science Publishers, New York, 1961. MR0141845 (25:5242)

[3] G. Browning and H.-O. Kreiss. Splitting methods for problems with different timescales. Monthly Weather Review, 122(11):2614-2622, 1994.

[4] Germund G. Dahlquist, Werner Liniger, and Olavi Nevanlinna. Stability of two-step methods for variable integration steps. SIAM J. Numer. Anal., 20(5):1071-1085, 1983. MR0714701 (85b:65079)

[5] Weinan E. Analysis of the heterogeneous multiscale method for ordinary differential equations. Commun. Math. Sci., 1(3):423-436, 2003. MR2069938

[6] Weinan E and Bjorn Engquist. The heterogeneous multiscale methods. Commun. Math. Sci., 1(1):87-132, 2003. MR1979846 (2004b:35019) 
[7] Weinan E, Di Liu, and Eric Vanden-Eijnden. Analysis of multiscale techniques for stochastic dynamical systems.

[8] Weinan E and Eric Vanden-Eijnden. Numerical techniques for multiscale dynamical systems with stochastic effects. Comm. Math. Sci., 1(2):385-391, 2003. MR.1980483

[9] B. García-Archilla, J. M. Sanz-Serna, and R. D. Skeel. Long-time-step methods for oscillatory differential equations. SIAM J. Sci. Comput., 20(3):930-963 (electronic), 1999. MR1648882 (99g:65087)

[10] C. W. Gear and D. R. Wells. Multirate linear multistep methods. BIT, 24(4):484-502, 1984. MR0764821 (86a:65057)

[11] C.W. Gear and Iaonnis G. Kevrekidis. "Coarse" integration/bifurcation analysis via microscopic simulators: micro-Galerkin methods. 2002.

[12] O. F. Graf and D. G. Bettis. Modified multirevolution integration methods for satellite orbit computation. Celestial Mech., 11:433-448, 1975. MR0373409(51:9609)

[13] E. Hairer and G. Wanner. Solving ordinary differential equations. II, volume 14 of Springer Series in Computational Mathematics. Springer-Verlag, Berlin, second edition, 1996. Stiff and differential-algebraic problems. MR.1439506 (97m:65007)

[14] Ernst Hairer, Christian Lubich, and Gerhard Wanner. Geometric numerical integration, volume 31 of Springer Series in Computational Mathematics. Springer-Verlag, Berlin, 2002. Structure-preserving algorithms for ordinary differential equations. MR 1904823 (2003f:65203)

[15] Arieh Iserles. On the global error of discretization methods for highly-oscillatory ordinary differential equations. BIT, 42(3):561-599, 2002. MR.1931887(2003i:65056)

[16] Tosio Kato. Perturbation theory for linear operators. Classics in Mathematics. SpringerVerlag, Berlin, 1995. Reprint of the 1980 edition. MR1335452 (96a:47025)

[17] N. Kopell. Invariant manifolds and the initialization problem for some atmospheric equations. Phys. D, 14(2):203-215, 1985. MR0784954 (86g:86005)

[18] Heinz-Otto Kreiss. Difference methods for stiff ordinary differential equations II. Preprint.

[19] Heinz-Otto Kreiss. Problems with different time scales for ordinary differential equations. SIAM J. Numer. Anal., 16(6):980-998, 1979. MR0551320 (81a:65087)

[20] Heinz-Otto Kreiss and Jens Lorenz. Manifolds of slow solutions for highly oscillatory problems. Indiana Univ. Math. J., 42(4):1169-1191, 1993. MR.1266089 (95f:34077)

[21] V. I. Lebedev and S. A. Finogenov. The use of ordered Cebyšev parameters in iteration methods. Ž. Vyčisl. Mat. i Mat. Fiz., 16(4):895-907, 1084, 1976. MR0443314(56:1684)

[22] Ben Leimkuhler and Sebastian Reich. A reversible averaging integrator for multiple time-scale dynamics. J. Comput. Phys., 171(1):95-114, 2001. MR1843642 (2002d:65064)

[23] Di Liu. Topics in the analysis and computation of stochastic differential equations. Ph.D. thesis, Princeton University, 2003.

[24] David Mace and L. H. Thomas. An extrapolation formula for stepping the calculation of the orbit of an artificial satellite several revolutions ahead at a time. Astronom. J., 65:300-303, 1960. MR0119998 (22:10755)

[25] George Majda. Filtering techniques for systems of stiff ordinary differential equations. I. SIAM J. Numer. Anal., 21(3):535-566, 1984. MR0744172 (85i:65089)

[26] George Majda. Filtering techniques for systems of stiff ordinary differential equations. II. Error estimates. SIAM J. Numer. Anal., 22(6):1116-1134, 1985. MR0811187 (87d:65074)

[27] W. L. Miranker and G. Wahba. An averaging method for the stiff highly oscillatory problem. Math. Comp., 30(135):383-399, 1976. MR0423817 (54:11791)

[28] Linda R. Petzold. An efficient numerical method for highly oscillatory ordinary differential equations. SIAM J. Numer. Anal., 18(3):455-479, 1981. MR0615526 (82h:65059)

[29] Linda R. Petzold, Laurent O. Jay, and Jeng Yen. Numerical solution of highly oscillatory ordinary differential equations. In Acta numerica, 1997, volume 6 of Acta Numer., pages 437-483. Cambridge Univ. Press, Cambridge, 1997. MR1489260 (98k:65040)

[30] Robert E. Scheid, Jr. The accurate numerical solution of highly oscillatory ordinary differential equations. Math. Comp., 41(164):487-509, 1983. MR0717698(85i:65091)

[31] Robert E. Scheid, Jr. Difference methods for problems with different time scales. Math. Comp., 44(169):81-92, 1985. MR0771032 (86e:65098)

[32] B. Engquist, P. Lötstedt, and O. Runborg (Eds.). Lecture Notes in Computational Science and Engineering, Vol. 44, Springer, 2005. 
[33] Michael Alan Zeitzew. Numerical Methods for Nonlinear Ordinary Differential Equations with Different Time-Scales. Ph.D. thesis, UCLA, 1995.

[34] Abraham Ziv and Vardy Amdursky. On the numerical solution of stiff linear systems of the oscillatory type. SIAM J. Appl. Math., 33(4):593-606, 1977. MR0455417 (56:13655)

Department of Mathematics and PaCm, Princeton University, Princeton, New JerSey 08544, and Royal Institute of Technology (KTH), SE-100 44 Stockholm, Sweden

Institute for Advanced Study and Department of Mathematics, Princeton UniverSity, Princeton, New Jersey 08544

E-mail address: ytsai@math.princeton.edu 